\title{
UZAWA SMOOTHER IN MULTIGRID FOR THE COUPLED POROUS MEDIUM AND STOKES FLOW SYSTEM*
}

\author{
PEIYAO LUO ${ }^{\dagger}$, CARMEN RODRIGO $\ddagger$, FRANCISCO J. GASPAR ${ }^{\S}$, AND \\ CORNELIS W. OOSTERLEE
}

\begin{abstract}
The multigrid solution of coupled porous media and Stokes flow problems is considered. The Darcy equation as the saturated porous medium model is coupled to the Stokes equations by means of appropriate interface conditions. We focus on an efficient multigrid solution technique for the coupled problem, which is discretized by finite volumes on staggered grids, giving rise to a saddle point linear system. Special treatment is required regarding the discretization at the interface. An Uzawa smoother is employed in multigrid, which is a decoupled procedure based on symmetric Gauss-Seidel smoothing for velocity components and a simple Richardson iteration for the pressure field. Since a relaxation parameter is part of a Richardson iteration, local Fourier analysis is applied to determine the optimal parameters. Highly satisfactory multigrid convergence is reported, and, moreover, the algorithm performs very well for small values of the hydraulic conductivity and fluid viscosity, which are relevant for applications.
\end{abstract}

Key words. Darcy equation, porous medium, Stokes equation, free flow, coupling, interface conditions, multigrid method, Uzawa smoother, local Fourier analysis

AMS subject classifications. $65 \mathrm{~N} 55,65 \mathrm{~F} 10$

DOI. $10.1137 / 16 \mathrm{M} 1076514$

1. Introduction. Coupling of free flow and a saturated porous medium has received considerable attention due to applications in environmental and industrial contexts, such as in flood simulation, filtration, and contamination. It is challenging to deal with a coupled multiphysics system, since each part of the system is based on a different model, and an appropriate coupling at the interface is required. Flow in the saturated porous medium is modeled by the conventional Darcy equation here (the solid framework is assumed to be rigid, and there is no interaction between the fluid and the solid matrix in the porous medium), while the Newtonian flow through a channel is modeled by the incompressible Stokes equations. Appropriate interface conditions are based on the principles of mass conservation, equilibrium of normal stresses across the interface, and a special condition called the BeaversJoseph-Saffman condition [29, 42] describing the relation between the shear stress and the tangential velocity. Many researchers have studied multiphysics problems theoretically; see, for example, [2, 13, 33, 27].

The numerical solution of these multiphysics problems is also an active research

*Received by the editors May 23, 2016; accepted for publication (in revised form) April 12, 2017; published electronically October 26, 2017.

http://www.siam.org/journals/sisc/39-5/M107651.html

Funding: The first author's work was supported by the China Scholarship Council (CSC). The second author's research was partially supported by FEDER/MCYT Projects MTM2013-40842-P and the DGA (Grupo consolidado PDIE). The third and fourth authors received funding from the European Union's Horizon 2020 research and innovation programme under the Marie SklodowskaCurie grant agreement 705402, POROSOS.

†DIAM, Delft University of Technology, 2628CD Delft, The Netherlands (p.luo@tudelft.nl).

${ }^{\ddagger}$ Department of Applied Mathematics, University of Zaragoza, 50009 Zaragoza, Spain (carmenr@ unizar.es).

${ }^{\S} \mathrm{CWI}$, Centrum Wiskunde and Informatica, 1098 XG Amsterdam, The Netherlands (gaspar@ cwi.nl).

${ }^{\top}$ CWI, Centrum Wiskunde and Informatica, 1098 XG Amsterdam, The Netherlands, and DIAM, Delft University of Technology, 2628CD Delft, The Netherlands (c.w.oosterlee@cwi.nl).

S633 
area $[17,19,26,40,41]$. We discretize the Darcy-Stokes problem by the finite volume method on a staggered grid, which results in a symmetric system. The mixed formulation of the Darcy problem is used, so that the discretized equations on the staggered grid result in a matrix of saddle point form [4], where a zero block appears on the diagonal of the system matrix. The zero block thus appears in the matrix of the complete problem, because a stable staggered discretization is used for the Darcy-Stokes equations.

There are basically two ways to solve a coupled multiphysics system. A popular approach is based on the domain decomposition method (DDM) [39, 44]. DDM exploits the principle of divide-and-conquer and is based on decoupling the global problem so that mainly independent subproblems are to be solved. Several advanced iterative solvers of this type have been applied to the Darcy-Stokes system, for example, the Dirichlet-Neumann-type DDM [18], Robin-Robin DDM [14, 20], Lagrange multiplier-based DDM [32], and many others [11, 12, 36]. DDM is often used as a preconditioner for a Krylov subspace method for such coupled multiphysics problems. On the other hand, the so-called monolithic solution approach focuses on the simultaneous solution of the multiphysics system. Methods in this class typically exhibit robust convergence when there is a strong coupling between the two subsystems. Based on this insight, different monolithic methods have been proposed in the literature, such as preconditioned GMRES methods $[10,15,16]$, where it was demonstrated that block-triangular and constraint preconditioners yield mesh-independent convergence.

We solve the coupled system by developing an efficient monolithic multigrid algorithm. Often, multigrid methods have been applied for the efficient solution of saddle point systems, even dating back to the early days of multigrid for systems of incompressible Navier-Stokes equations [8,9]. Other efficient multigrid methods for Stokes and incompressible Navier-Stokes problems have been developed, for example, in $[5,23,28,31,46]$. In [1] an efficient monolithic method was proposed for the magneto-hydrodynamics system.

Within a multigrid method, the choice of the smoothing method plays an important role. Basically, there are two major categories of smoothers for saddle point problems, classified as coupled and decoupled smoothers; see [21, 22, 37]. Coupled smoothers, such as the Vanka smoother [46], or box relaxation, are governed by the fact that all equations of the system are updated simultaneously in a coupled fashion. Decoupled, equationwise, smoothing methods, however, are also popular, because of their convenient implementation. In this paper, we consider an equationwise smoother within monolithic multigrid for the Darcy-Stokes system. The Uzawa smoother [35] will be applied for this discrete coupled system. This smoother has been enhanced for the Stokes equations in [23]. For the problem considered here, the velocities in the Darcy and Stokes equations are updated first, after which the pressures for both subsystems are relaxed. The Uzawa smoother is based on a Richardson iteration in which a relaxation parameter is present. Local Fourier analysis (LFA) is applied to choose suitable values for the relaxation parameter. LFA is a powerful tool for the quantitative analysis of the convergence of multigrid, introduced by Brandt [6] in 1977 and then further developed in [7]. A general introduction can be found in [45], and software is available in [47]. As the optimal relaxation parameter for the Stokes problem has already been determined in [23], in the present paper we are concerned with the selection of an optimal parameter for the Darcy problem through LFA. Here, LFA is also used to confirm the convergence obtained from the monolithic multigrid method. LFA is applied to the Darcy and Stokes subproblems separately, and it is 
shown that the worst of these factors proves to be the global multigrid convergence for the coupled problem. By using the monolithic multigrid approach, we are able to achieve a textbook multigrid convergence rate, even when the values of the physical parameters are realistically small.

We deal with the so-called multiblock multigrid method which is based on the grid partitioning technique [45]. Boundary updates are communicated between neighboring blocks within the algorithm on each multigrid level. In [45, Chapter 6], a detailed introduction of the grid partitioning technique is given. The convergence of the multiblock multigrid algorithm is identical to its single block equivalent in our case.

The paper is organized as follows. The equations in free flow and porous media, together with the interface conditions, are introduced in section 2. Section 3 deals with the discretization of the coupled Darcy-Stokes system. We give the discrete formulas for the coupled system including the discretization at the interface. The solution method, the Uzawa smoother, and its analysis by means of LFA are presented in section 4. In section 5, several numerical experiments are performed to show the efficiency of the algorithm. Conclusions are drawn in section 6 .

2. Problem formulation. In this work, we restrict ourselves to the two-dimensional Darcy-Stokes problem. The proposed solution strategy can be straightforwardly extended to a three-dimensional setting.

We consider the Darcy-Stokes problem on a bounded domain $\Omega \subset \mathbb{R}^{2}$ and assume that $\Omega$ is subdivided into two disjoint subdomains $\Omega^{d}$ and $\Omega^{f}$, corresponding to the porous medium and free-flow regions, respectively. Let $\Gamma$ denote the interface between the two subregions, that is, $\Gamma=\partial \Omega^{d} \cap \partial \Omega^{f}$. The geometry of the problem is represented in Figure 1, where we also display $\mathbf{n}^{f}$ and $\mathbf{n}^{d}$, denoting the unit outward normal vectors on $\partial \Omega^{f}$ and $\partial \Omega^{d}$, respectively. At the interface $\Gamma$, we have $\mathbf{n}^{f}=-\mathbf{n}^{d}$.

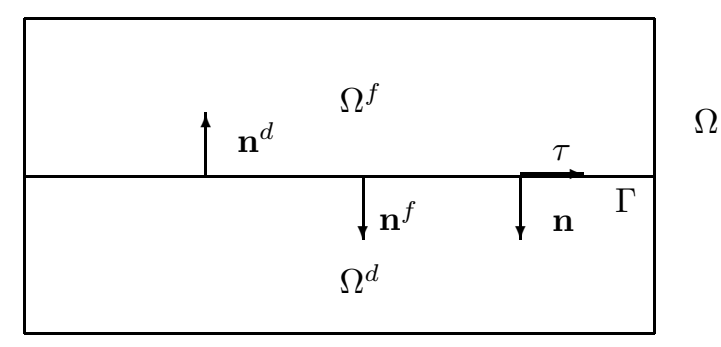

FIG. 1. Geometry of the Darcy-Stokes problem. Subdivision of the domain $\Omega$ into a free-flow subregion $\Omega^{f}$ and a porous medium subdomain $\Omega^{d}$ by an internal interface $\Gamma$.

We describe the porous medium and free-flow models considered on the different subdomains, including the boundary conditions for the outer boundaries, in sections 2.1 and 2.2, and the internal interface conditions governing the interactions between the fluid and the porous medium in section 2.3.

2.1. Porous medium description. The fluid flow through a rigid and saturated porous medium $\Omega^{d}$ is described by Darcy's law, which is an expression of conservation of momentum. The mixed formulation of the Darcy problem is natural for computations in the porous medium region since it allows one to directly approximate the velocity. In this work, we will consider this formulation, which reads

$$
\begin{array}{rc}
\mathbb{K}^{-1} \mathbf{u}^{d}+\nabla p^{d}=\mathbf{0} & \text { in } \Omega^{d}, \\
\nabla \cdot \mathbf{u}^{d}=f^{d} & \text { in } \Omega^{d},
\end{array}
$$


where $\mathbf{u}^{d}=\left(u^{d}, v^{d}\right)$ describes the velocity and $p^{d}$ the fluid pressure inside the porous medium. $\mathbb{K}$ is the hydraulic conductivity tensor, representing the properties of the porous medium and the fluid. Here, the case $\mathbb{K}=K \mathbb{I}, K>0$, is considered. Sinks and sources are described by the force term $f^{d}$.

We assume that the boundary $\partial \Omega^{d} \backslash \Gamma$ is the union of two disjoint subsets $\Gamma_{D}^{d}$ and $\Gamma_{N}^{d}$, where Dirichlet and Neumann boundary conditions are imposed. More concretely, we consider the following boundary conditions:

$$
\begin{array}{rlrl}
p^{d} & =g_{D}^{d} & & \text { on } \Gamma_{D}^{d}, \\
\mathbf{u}^{d} \cdot \mathbf{n}^{d}=g_{N}^{d} & & \text { on } \Gamma_{N}^{d} .
\end{array}
$$

2.2. Free-flow description. The free-flow subproblem is modeled by using the Stokes equations for a viscous, incompressible, Newtonian fluid. It is a linearized form of the Navier-Stokes equations in the limit case when the nonlinear term becomes negligible. The motion of the Stokes flow in the region $\Omega^{f}$ is described by

$$
\begin{aligned}
-\nabla \cdot \boldsymbol{\sigma}^{f}=\mathbf{f}^{f} & \text { in } \Omega^{f}, \\
\nabla \cdot \mathbf{u}^{f}=0 & \text { in } \Omega^{f},
\end{aligned}
$$

where $\mathbf{u}^{f}=\left(u^{f}, v^{f}\right)$ is the fluid velocity, $\mathbf{f}^{f}=\left(f_{1}^{f}, f_{2}^{f}\right)$ represents a prescribed force, and the fluid stress tensor $\boldsymbol{\sigma}^{f}$ is given by

$$
\boldsymbol{\sigma}^{f}=-p^{f} \mathbf{I}+2 \nu \mathbf{D}\left(\mathbf{u}^{f}\right),
$$

with $p^{f}$ denoting the fluid pressure and $\nu$ representing the fluid viscosity and where $\mathbf{D}\left(\mathbf{u}^{f}\right)=\left(\nabla \mathbf{u}^{f}+\left(\nabla \mathbf{u}^{f}\right)^{T}\right) / 2$ is the strain tensor. The first equation in (3) is the so-called momentum equation, and the second one is the continuity equation. By writing the stress tensor $\boldsymbol{\sigma}^{f}$ as

$$
\boldsymbol{\sigma}^{f}:=\left(\begin{array}{cc}
\sigma_{x x} & \sigma_{x y} \\
\sigma_{y x} & \sigma_{y y}
\end{array}\right)
$$

where

$$
\sigma_{x x}=-p^{f}+2 \nu \frac{\partial u^{f}}{\partial x}, \quad \sigma_{x y}=\sigma_{y x}=\nu\left(\frac{\partial u^{f}}{\partial y}+\frac{\partial v^{f}}{\partial x}\right), \quad \sigma_{y y}=-p^{f}+2 \nu \frac{\partial v^{f}}{\partial y}
$$

we can rewrite (3) as follows:

$$
\begin{aligned}
-\nu\left(\frac{\partial^{2} u^{f}}{\partial x^{2}}+\frac{\partial^{2} u^{f}}{\partial y^{2}}\right)+\frac{\partial p^{f}}{\partial x} & =f_{1}^{f} \text { in } \Omega^{f}, \\
-\nu\left(\frac{\partial^{2} v^{f}}{\partial x^{2}}+\frac{\partial^{2} v^{f}}{\partial y^{2}}\right)+\frac{\partial p^{f}}{\partial y} & =f_{2}^{f} \text { in } \Omega^{f}, \\
\frac{\partial u^{f}}{\partial x}+\frac{\partial v^{f}}{\partial y} & =0 \text { in } \Omega^{f} .
\end{aligned}
$$

For the free-flow subproblem, we split $\partial \Omega^{f} \backslash \Gamma$ into two disjoint parts $\Gamma_{D}^{f}$ and $\Gamma_{N}^{f}$, where we impose the following boundary conditions:

$$
\begin{aligned}
\mathbf{u}^{f} & =\mathbf{g}_{D}^{f} & & \text { on } \Gamma_{D}^{f}, \\
\boldsymbol{\sigma}^{f} \cdot \mathbf{n}^{f} & =\mathbf{g}_{N}^{f} & & \text { on } \Gamma_{N}^{f} .
\end{aligned}
$$

Copyright $@$ by SIAM. Unauthorized reproduction of this article is prohibited. 
2.3. Interface conditions. The Darcy and Stokes systems must be coupled across the internal interface $\Gamma$ by adequate conditions. To describe them, we fix the normal vector to the interface to be $\mathbf{n}=\mathbf{n}^{f}=-\mathbf{n}^{d}$, and we denote $\boldsymbol{\tau}$ (see Figure 1) as the tangential unit vector at the interface $\Gamma$, obtained by rotating the normal vector in the counterclockwise direction by $90^{\circ}$. Across $\Gamma$ the continuity of fluxes and normal stresses must be imposed. This gives rise to the following two standard coupling conditions on $\Gamma$ :

- Mass conservation:

$$
\mathbf{u}^{f} \cdot \mathbf{n}=\mathbf{u}^{d} \cdot \mathbf{n} \quad \text { on } \Gamma .
$$

- Balance of normal stresses:

$$
-\mathbf{n} \cdot \boldsymbol{\sigma}^{f} \cdot \mathbf{n}=p^{d} \quad \text { on } \Gamma .
$$

As the third coupling condition, the so-called Beavers-Joseph-Saffman interface condition is widely used, which is supported by experimental findings and rigorous mathematical theory of homogenization. This condition relates the tangential velocity along the interface with the fluid stresses, that is,

$$
\alpha \mathbf{u}^{f} \cdot \boldsymbol{\tau}+\boldsymbol{\tau} \cdot \boldsymbol{\sigma}^{f} \cdot \mathbf{n}=0 \quad \text { on } \Gamma,
$$

where $\alpha$ is a parameter which needs to be experimentally determined and depends on the properties of the porous medium.

An alternative to this third interface condition neglects the second term in (12), giving rise to a no-slip interface condition,

$$
\mathbf{u}^{f} \cdot \boldsymbol{\tau}=0 \quad \text { on } \Gamma .
$$

3. Discretization. The finite volume method on a staggered grid [38] is considered as the discretization scheme for the Darcy-Stokes problem. By using this discretization we ensure that spurious oscillations do not appear in the numerical solution [25], and we obtain a mass conservative algorithm for the whole system. The computational domain is partitioned into square blocks of size $h \times h$, so that the grid is conforming at the interface $\Gamma$. For notational convenience, we choose equalsized blocks, but the description in the more general case would be straightforward. Different control volumes are defined depending on which variable is considered. In Figure 2, we represent in different colors the control volumes corresponding to the primary variables $u, v$, and $p .^{1}$ The pressure unknowns $p$ are defined at the centers of the blocks (marked by $\times$-points in Figure 2), and the components of the velocity unknowns, $u$ and $v$, are located at the centers of the block faces (denoted by the oand $\bullet$-points in the same figure). For the description of the discrete scheme, we need to fix an adequate indexing for the unknowns, which can be seen in Figure 3, where each unknown is depicted together with the corresponding control volume and the different variables around it.

We describe in detail the discretization for the mixed formulation of the Darcy problem in section 3.1, the discrete scheme for the Stokes equations in section 3.2, and the special discretization considered for the internal interface $\Gamma$ in section 3.3.

\footnotetext{
${ }^{1}$ In the following figures, the superscript $d / f$ is omitted, as we have the same arrangement for both subproblems.
}

Copyright $\odot$ by SIAM. Unauthorized reproduction of this article is prohibited. 


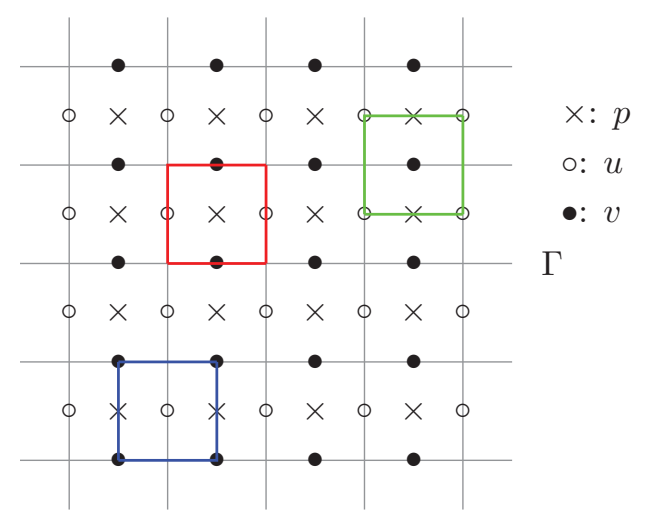

FIG. 2. Staggered grid location of unknowns for the coupled model, and corresponding control volumes.

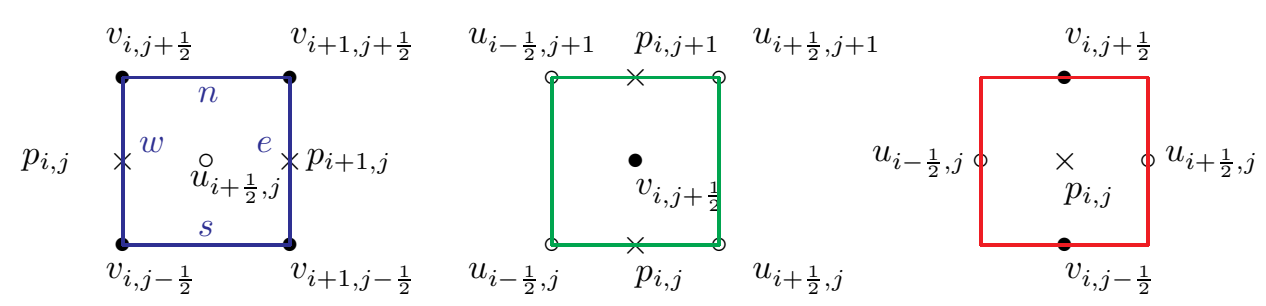

FIG. 3. Control volumes for the primary unknowns: $u$ (left), $v$ (middle), $p$ (right), together with the corresponding indexing for each variable.

3.1. Discretization of Darcy equations. Since in the mixed formulation of the Darcy problem the pressure and the velocities are the primary variables, we describe the corresponding discretizations for each equation. Regarding the horizontal velocity unknown, let us consider control volume $V_{i+1 / 2, j}$ for variable $u_{i+1 / 2, j}^{d}$ (Figure 3 (left)). By discretizing the first equation in (1) over such a control volume, we obtain

$$
K^{-1} u_{i+\frac{1}{2}, j}^{d}+\frac{p_{i+1, j}^{d}-p_{i, j}^{d}}{h}=0
$$

Similarly, by discretizing the equation for $v^{d}$ in (1) over a control volume $V_{i, j+1 / 2}$ (Figure 3 (middle)), one obtains the discrete equation for the vertical velocity unknown $v_{i, j+1 / 2}^{d}$,

$$
K^{-1} v_{i, j+\frac{1}{2}}^{d}+\frac{p_{i, j+1}^{d}-p_{i, j}^{d}}{h}=0
$$

Equations (14) and (15) are associated with internal velocity unknowns. In the case of variables located at the external boundary where Dirichlet boundary conditions for the pressure are imposed, the corresponding control volumes to consider are half the size of the inner control volumes and are treated accordingly.

Finally, the discrete equation corresponding to the pressure unknown $p_{i, j}^{d}$ is obtained by discretizing the second equation in (1) over control volume $V_{i, j}$ (Figure 3 
(right)), resulting in

$$
\frac{u_{i+\frac{1}{2}, j}^{d}-u_{i-\frac{1}{2}, j}^{d}}{h}+\frac{v_{i, j+\frac{1}{2}}^{d}-v_{i, j-\frac{1}{2}}^{d}}{h}=f_{i, j}^{d} .
$$

3.2. Discretization of Stokes equations. We proceed by briefly presenting the discretization of the Stokes equations. Regarding the mass balance equation, similarly as in the previous section, we discretize the second equation in (3) over control volume $V_{i, j}$ to obtain the following discrete equation:

$$
\frac{u_{i+\frac{1}{2}, j}^{f}-u_{i-\frac{1}{2}, j}^{f}}{h}+\frac{v_{i, j+\frac{1}{2}}^{f}-v_{i, j-\frac{1}{2}}^{f}}{h}=0 .
$$

Regarding the momentum equation in (3), we describe only the first component of the equation since the second one would be deduced in a similar way. Thus, discretizing such a component over control volume $V_{i+\frac{1}{2}, j}$ yields

$$
-\left(\frac{\left(\sigma_{x x}\right)_{e}-\left(\sigma_{x x}\right)_{w}}{h}+\frac{\left(\sigma_{x y}\right)_{n}-\left(\sigma_{x y}\right)_{s}}{h}\right)=\left(f_{1}^{f}\right)_{i+\frac{1}{2}, j},
$$

where $\sigma_{x x}$ and $\sigma_{x y}$ are components of the stress tensor. Approximating these components as

$$
\begin{aligned}
\left(\sigma_{x x}\right)_{e} & =-p_{i+1, j}^{f}+2 \nu \frac{u_{i+\frac{3}{2}, j}^{f}-u_{i+\frac{1}{2}, j}^{f}}{h}, \\
\left(\sigma_{x y}\right)_{n} & =\nu\left(\frac{u_{i+\frac{1}{2}, j+1}^{f}-u_{i+\frac{1}{2}, j}^{f}}{h}+\frac{v_{i+1, j+\frac{1}{2}}^{f}-v_{i, j+\frac{1}{2}}^{f}}{h}\right), \\
\left(\sigma_{x x}\right)_{w} & =-p_{i, j}^{f}+2 \nu \frac{u_{i+\frac{1}{2}, j}^{f}-u_{i-\frac{1}{2}, j}^{f}}{h}, \\
\left(\sigma_{x y}\right)_{s} & =\nu\left(\frac{u_{i+\frac{1}{2}, j}^{f}-u_{i+\frac{1}{2}, j-1}^{f}}{h}+\frac{v_{i+1, j-\frac{1}{2}}^{f}-v_{i, j-\frac{1}{2}}^{f}}{h}\right),
\end{aligned}
$$

and substituting them in (18), we obtain the following equation:

$$
\begin{gathered}
-\frac{2 \nu}{h^{2}}\left(u_{i+\frac{3}{2}, j}^{f}-2 u_{i+\frac{1}{2}, j}^{f}+u_{i-\frac{1}{2}, j}^{f}\right)-\frac{\nu}{h^{2}}\left(u_{i+\frac{1}{2}, j+1}^{f}-2 u_{i+\frac{1}{2}, j}^{f}+u_{i+\frac{1}{2}, j-1}^{f}\right) \\
-\frac{\nu}{h^{2}}\left(v_{i+1, j+\frac{1}{2}}^{f}-v_{i, j+\frac{1}{2}}^{f}-v_{i+1, j-\frac{1}{2}}^{f}+v_{i, j-\frac{1}{2}}^{f}\right)+\frac{1}{h}\left(p_{i+1, j}^{f}-p_{i, j}^{f}\right)=\left(f_{1}^{f}\right)_{i+\frac{1}{2}, j} .
\end{gathered}
$$

3.3. Discretization of the interface. In this section, we describe how we deal with the interface conditions. Our proposal is to obtain a special discrete equation for the unknowns at the internal interface. Due to the staggered arrangement of the unknowns, the only variables at the interface are the vertical components of the velocity; see Figure 4. For this purpose, we integrate the momentum equation of the Stokes system over a half-volume, as displayed in red in Figure 4, giving rise to the following equation:

$$
-\left(\frac{\left(\sigma_{x y}\right)_{e}-\left(\sigma_{x y}\right)_{w}}{h}+\frac{\left(\sigma_{y y}\right)_{n}-\left(\sigma_{y y}\right)_{s}}{h / 2}\right)=\left(f_{2}^{f}\right)_{i, j+\frac{1}{2}}
$$

Copyright $@$ by SIAM. Unauthorized reproduction of this article is prohibited. 


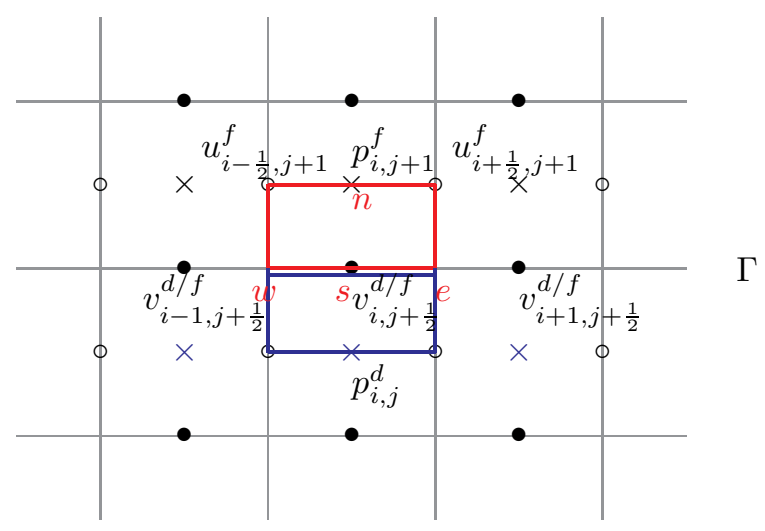

FIG. 4. Staggered grid location of the unknowns for the interface conditions.

where, as can be seen in Figure $4, e$ and $w$ denote locations at the interface, whereas $n$ and $s$ denote the locations of $p_{i, j+1}^{f}$ and $v_{i, j+\frac{1}{2}}^{d / f}$, respectively. The approximation of $\left(\sigma_{y y}\right)_{n}$ is easily obtained as

$$
\left(\sigma_{y y}\right)_{n}=-p_{i, j+1}^{f}+\frac{2 \nu}{h}\left(v_{i, j+\frac{3}{2}}^{f}-v_{i, j+\frac{1}{2}}^{f}\right),
$$

whereas the approximation of the other components of the stress tensor requires the use of the interface conditions. To approximate the component $\left(\sigma_{y y}\right)_{s}$, we directly apply the interface condition (11), obtaining

$$
\left(\sigma_{y y}\right)_{s}=-p_{s}^{d} .
$$

The pressure $p_{s}^{d}$ is not known at the interface, but it can be approximated with the help of the Darcy problem. By integrating the corresponding equation over a halfvolume as displayed in blue in Figure 4, we obtain

$$
K^{-1} v_{i, j+\frac{1}{2}}^{d}+\frac{p_{s}^{d}-p_{i, j}^{d}}{h / 2}=0
$$

Substituting this equation in (21), the approximation reads

$$
\left(\sigma_{y y}\right)_{s}=-p_{i, j}^{d}+\frac{h}{2 K} v_{i, j+\frac{1}{2}}^{d} .
$$

To approximate the remaining components of the stress tensor, we need to use either the no-slip or the Beavers-Joseph-Saffman interface condition. Here, we consider the latter since it is the most involved case. The standard approximation of the BeaversJoseph-Saffman condition (12) at the location denoted by $e$ reads

$$
\alpha u_{e}^{f}-\nu\left(\frac{u_{i+\frac{1}{2}, j+1}^{f}-u_{e}^{f}}{h / 2}+\frac{v_{i+1, j+\frac{1}{2}}^{f}-v_{i, j+\frac{1}{2}}^{f}}{h}\right)=0 .
$$

Here, $u_{e}^{f}$ can be obtained from (24) and substituted into the standard approximation 
of the stress $\left(\sigma_{x y}\right)_{e}$, resulting in

$$
\begin{aligned}
\left(\sigma_{x y}\right)_{e} & =\nu\left(\frac{u_{i+\frac{1}{2}, j+1}^{f}-u_{e}^{f}}{h / 2}+\frac{v_{i+1, j+\frac{1}{2}}^{f}-v_{i, j+\frac{1}{2}}^{f}}{h}\right) \\
& =\frac{2 \nu m}{h} u_{i+\frac{1}{2}, j+1}^{f}+\nu m \frac{v_{i+1, j+\frac{1}{2}}^{f}-v_{i, j+\frac{1}{2}}^{f}}{h},
\end{aligned}
$$

where $m=\left(1-\frac{2 \nu}{h \alpha+2 \nu}\right)$. The approximation of $\left(\sigma_{x y}\right)_{w}$ can be calculated in a similar way. The discrete equation for the vertical velocities for the Stokes problem at the interface is thus obtained by substituting (20), (23), and (25) into (19), giving

$$
\begin{aligned}
- & \frac{2 \nu m}{h^{2}}\left(u_{i+\frac{1}{2}, j+1}^{f}-u_{i-\frac{1}{2}, j+1}^{f}\right)-\frac{\nu m}{h^{2}}\left(v_{i+1, j+\frac{1}{2}}^{f}-2 v_{i, j+\frac{1}{2}}^{f}+v_{i-1, j+\frac{1}{2}}^{f}\right) \\
& +\frac{2}{h}\left(p_{i, j+1}^{f}-p_{i, j}^{d}\right)-\frac{4 \nu}{h^{2}}\left(v_{i, j+\frac{3}{2}}^{f}-v_{i, j+\frac{1}{2}}^{f}\right)+\frac{1}{K} v_{i, j+\frac{1}{2}}^{f}=\left(f_{2}^{f}\right)_{i, j+\frac{1}{2}},
\end{aligned}
$$

where we have used the interface condition $v_{i, j+\frac{1}{2}}^{d}=v_{i, j+\frac{1}{2}}^{f}$.

The discretization at the interface is of great importance and can be viewed as a relevant ingredient toward the construction of a highly efficient multigrid method. Since the coupled system is treated as a single problem, the equations of fluid dynamics, solid mechanics, and their complex interaction are all included in one discrete formulation. By such a discretization, the fully coupled system possesses a saddle point structure which is suitable for monolithic multigrid.

4. Numerical method. This section is devoted to the design of a monolithic geometric multigrid for the Darcy-Stokes problem. For this purpose, we will first study the application of multigrid methods based on Uzawa smoothers to the Darcy and Stokes problems separately. In this analysis we will take into account the development of an LFA technique to obtain suitable parameters for these methods. These algorithms will form the basis for constructing a monolithic multigrid for the coupled problem. This will be possible since the individual Stokes and Darcy systems, as well as the fully coupled problem, lead to saddle point linear systems of the form

$$
\left(\begin{array}{cc}
A & B^{T} \\
B & 0
\end{array}\right)\left(\begin{array}{l}
\mathbf{u} \\
p
\end{array}\right)=\left(\begin{array}{l}
\mathbf{g} \\
f
\end{array}\right)
$$

by choosing an adequate arrangement of the unknowns. For both problems, $B^{T}$ and $B$ represent the discrete gradient and the minus discrete divergence operators, respectively, and $A$ is the discrete representation of either the Laplace-type operator $-\nu \Delta$ for the Stokes equations, or $K^{-1} I$ for the Darcy equation. For the coupled problem, rearranging the vector of unknowns to order first the velocities for both problems and thereafter the pressure unknowns, we obtain the following linear system:

$$
\left(\begin{array}{cccc}
A^{d} & 0 & \left(B^{d}\right)^{T} & 0 \\
0 & A^{f} & R & \left(B^{f}\right)^{T} \\
B^{d} & R & 0 & 0 \\
0 & B^{f} & 0 & 0
\end{array}\right)\left(\begin{array}{c}
\mathbf{u}^{d} \\
\mathbf{u}^{f} \\
p^{d} \\
p^{f}
\end{array}\right)=\left(\begin{array}{c}
\mathbf{0} \\
\mathbf{f}^{f} \\
f^{d} \\
0
\end{array}\right),
$$

where the system matrix in (28) has the same saddle point structure as in (27), by denoting

$$
A=\left(\begin{array}{cc}
A^{d} & 0 \\
0 & A^{f}
\end{array}\right), \quad B=\left(\begin{array}{cc}
B^{d} & R \\
0 & B^{f}
\end{array}\right), \quad B^{T}=\left(\begin{array}{cc}
\left(B^{d}\right)^{T} & 0 \\
R & \left(B^{f}\right)^{T}
\end{array}\right) .
$$


Here, $R$ is a diagonal matrix containing the relations given by formula (26) between the vertical velocities $v^{f}$ and the corresponding pressure unknowns in the Darcy domain $p^{d}$. So, most of its elements are zero, and the only nonzero diagonal terms are those corresponding to the Darcy pressure unknowns close to the internal interface, appearing in the equations of the vertical velocities on $\Gamma$. Due to this structure of the coupled problem, a geometric multigrid method together with an Uzawa smoother, which will be introduced in section 4.1, can be applied for the whole system. As we will see, the choice of adequate relaxation parameters for the Uzawa smoother on each subproblem will be crucial for excellent multigrid convergence.

4.1. Multigrid based on Uzawa smoother. In order to develop an efficient multigrid solver, it is necessary to carefully consider each component of multigrid, such as the smoothing operator and the coarse grid correction components (i.e., the restriction and prolongation operators, and the coarse grid operator). Regarding the coarse grid correction, geometric grid coarsening is chosen, as we will deal with regular Cartesian grids. The sequence of coarse grids is obtained by doubling the mesh size in each spatial direction. We further use well-known, proven components for the transfer operators between the fine and coarse grids, which are dictated by the staggered grid arrangement, and focus our efforts on the analysis of the smoothing operator. In particular, the interplay between the relaxation method (the smoother) and the coarse grid correction is crucial for the multigrid performance.

Taking the staggered arrangement of the unknowns into account, the intergrid transfer operators that act on the different unknowns are defined as follows: at velocity grid points six-point restrictions are considered, and at pressure grid points a fourpoint restriction is applied. In stencil notation, the restriction operators are given by

$$
R_{h, 2 h}^{u}=\frac{1}{8}\left(\begin{array}{rrr}
1 & 2 & 1 \\
& * & \\
1 & 2 & 1
\end{array}\right)_{h}, R_{h, 2 h}^{v}=\frac{1}{8}\left(\begin{array}{lll}
1 & & 1 \\
2 & * & 2 \\
1 & & 1
\end{array}\right)_{h}, R_{h, 2 h}^{p}=\frac{1}{4}\left(\begin{array}{ccc}
1 & & 1 \\
& * & \\
1 & & 1
\end{array}\right)_{h},
$$

respectively. As the prolongation operators $P_{2 h, h}^{u / v / p}$, we choose the adjoints of the restrictions.

The choice of smoother requires special attention due to the saddle point structure of the considered system. An Uzawa smoother, which was proposed for the Stokes problem in [23], is considered for the coupled system.

The Uzawa smoother. We give a general description of the considered Uzawa smoother. We will see that this relaxation can be successfully applied in multigrid for both Stokes and Darcy systems, and also in the multigrid method for the coupled system. The Uzawa smoother is obtained by splitting the discrete operator as follows:

$$
\left(\begin{array}{cc}
A & B^{T} \\
B & 0
\end{array}\right)=\left(\begin{array}{cc}
M_{A} & 0 \\
B & -\omega^{-1} I
\end{array}\right)-\left(\begin{array}{cc}
M_{A}-A & -B^{T} \\
0 & -\omega^{-1} I
\end{array}\right)
$$

where $M_{A}$ is a typical smoother for $A$ and $\omega$ is some positive parameter. $M_{A}$ makes the approach less costly because of the inexact solve for velocities at each iteration.

From a given approximation of the solution to the system $(\mathbf{u}, p)^{T}$, the relaxed approximation $(\widehat{\mathbf{u}}, \widehat{p})^{T}$ is computed according to the decoupled Uzawa smoother in the following way:

$$
\left(\begin{array}{cc}
M_{A} & 0 \\
B & -\omega^{-1} I
\end{array}\right)\left(\begin{array}{c}
\widehat{\mathbf{u}} \\
\widehat{p}
\end{array}\right)=\left(\begin{array}{cc}
M_{A}-A & -B^{T} \\
0 & -\omega^{-1} I
\end{array}\right)\left(\begin{array}{l}
\mathbf{u} \\
p
\end{array}\right)+\left(\begin{array}{l}
\mathbf{g} \\
f
\end{array}\right) .
$$


More concretely, a single step of the relaxation process is described as follows:

- Relax the velocities by applying $M_{A}: \hat{\mathbf{u}}=\mathbf{u}+M_{A}^{-1}\left(\mathrm{~g}-A \mathbf{u}-B^{T} p\right)$.

- Update the pressure: $\hat{p}=p+\omega(B \hat{\mathbf{u}}-f)$.

Notice that, in general, the Uzawa method is equivalent to a stationary Richardson iteration applied to the Schur complement system. This relation allows one to deduce an expression for parameter $\omega$ which minimizes the spectral radius of the corresponding iteration matrix, i.e.,

$$
\omega=\frac{2}{\lambda_{\max }+\lambda_{\min }},
$$

where $\lambda_{\max }$ and $\lambda_{\min }$ denote the largest and smallest eigenvalues of the Schur complement, respectively (see [4]). In the local Fourier analysis section we will estimate optimal relaxation parameter $\omega$ in the Uzawa smoother for the Darcy and Stokes problems, and we will also obtain a similar expression in which $\lambda_{\max }$ and $\lambda_{\min }$ are substituted for the largest and smallest eigenvalues but only on the high frequencies for smoothing analysis purposes.

An Uzawa smoother using two forward Gauss-Seidel sweeps as $M_{A}$ was suggested in [35] for the Stokes problem. In [23] another variant was considered, where $M_{A}$ was based on the symmetric Gauss-Seidel iterations for A; i.e.,

$$
M_{A}=\left(D_{A}+L_{A}\right) D_{A}^{-1}\left(D_{A}+U_{A}\right),
$$

where $D_{A}, L_{A}$, and $U_{A}$ are, respectively, the diagonal, the strictly lower, and the strictly upper parts of $A$. The symmetric Gauss-Seidel method consists of one forward and one backward sweep for all velocities in the computational domain. Numerical experiments in [23] revealed that, for essentially the same cost, the convergence associated with $M_{A}$ in (31) is most efficient. So, this variant is the one that we extend to the Darcy equation. The efficiency of the proposed Uzawa smoother for threedimensional Stokes and Biot poroelasticity equations was presented in [23] and [34], respectively.

In addition, $M_{A}$ has two important properties needed in our theoretical analysis, in addition to its efficiency as a smoother. One is that $M_{A}$ is symmetric positive definite (SPD) if $A$ is SPD. The other is that the associated largest eigenvalue satisfies (see, e.g., [3, Theorem 7.17]) $\lambda_{\max }\left(M_{A}^{-1} A\right) \leq 1$. We wish to mention the importance of the choice of an adequate value for the relaxation parameter $\omega$ to obtain a satisfactory performance of the Uzawa smoother. As in [23] for the Stokes equations, analytic expressions for $\omega$ can also be obtained by means of a theoretical analysis for the Darcy problem. We present this analysis first in a general way to make this work self-contained, and later we will describe the particular case of the Darcy equations.

4.2. LFA. We briefly introduce the LFA for staggered grids before we focus on the analysis of the Uzawa smoother.

Basis of LFA. To perform LFA, all discrete operators are assumed to be defined on an infinite grid $G_{h}$, and boundary conditions are neglected. Due to the arrangement of unknowns on a staggered grid, $G_{h}$ is divided into three subsets $G_{h}^{k}$ defined as

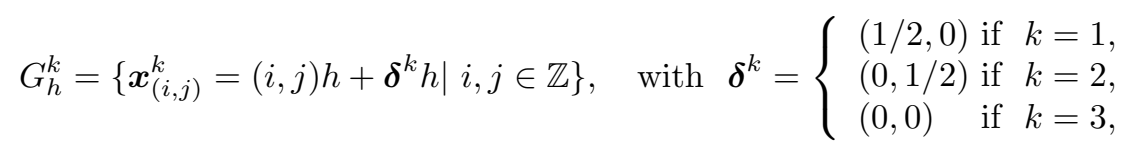

such that $G_{h}=G_{h}^{1} \cup G_{h}^{2} \cup G_{h}^{3}$. Corresponding to Figure 2, the velocities $u^{d / f}$ and $v^{d / f}$ are situated at nodes $\boldsymbol{x}_{i, j}^{1}$ and $\boldsymbol{x}_{i, j}^{2}$, respectively, whereas $\boldsymbol{x}_{i, j}^{3}$ is for the pressure 
unknowns $p^{d / f}$. The basic idea of LFA is that all occurring multigrid components, the discrete approximation, and its corresponding error or residual can be represented by formal linear combinations of Fourier modes. In the case of a staggered grid, considering $\boldsymbol{\varphi}_{h}^{1}\left(\boldsymbol{\theta}, \boldsymbol{x}_{i, j}\right)=\left(e^{i \boldsymbol{\theta} \cdot \boldsymbol{x}_{i, j}^{1} / h}, 0,0\right)^{T}, \boldsymbol{\varphi}_{h}^{2}\left(\boldsymbol{\theta}, \boldsymbol{x}_{i, j}\right)=\left(0, e^{\boldsymbol{i} \cdot \boldsymbol{x}_{i, j}^{2} / h}, 0\right)^{T}$, and $\boldsymbol{\varphi}_{h}^{3}\left(\boldsymbol{\theta}, \boldsymbol{x}_{i, j}\right)=\left(0,0, e^{i \boldsymbol{\theta} \cdot \boldsymbol{x}_{i, j}^{3} / h}\right)^{T}$, the Fourier modes are defined as

$$
\boldsymbol{\varphi}_{h}\left(\boldsymbol{\theta}, \boldsymbol{x}_{i, j}\right):=\left[\boldsymbol{\varphi}_{h}^{1}\left(\boldsymbol{\theta}, \boldsymbol{x}_{i, j}\right) \boldsymbol{\varphi}_{h}^{2}\left(\boldsymbol{\theta}, \boldsymbol{x}_{i, j}\right) \boldsymbol{\varphi}_{h}^{3}\left(\boldsymbol{\theta}, \boldsymbol{x}_{i, j}\right)\right],
$$

where $\boldsymbol{x}_{i, j}=\left(\boldsymbol{x}_{i, j}^{1}, \boldsymbol{x}_{i, j}^{2}, \boldsymbol{x}_{i, j}^{3}\right)$ and $\boldsymbol{\theta} \in \boldsymbol{\Theta}:=(-\pi, \pi]^{2}$, which form a unitary basis of the space of infinite grid functions. The Fourier space generated by Fourier modes is given by $\mathcal{F}\left(G_{h}\right):=\operatorname{span}\left\{\boldsymbol{\varphi}_{h}(\boldsymbol{\theta}, \cdot) \mid \boldsymbol{\theta} \in \boldsymbol{\Theta}\right\}$. For the analysis, we distinguish high- and low-frequency components on $G_{h}$,

$$
\Theta_{\text {low }}^{2 h}:=\left(-\frac{\pi}{2}, \frac{\pi}{2}\right]^{2}, \quad \Theta_{h i g h}^{2 h}:=\Theta \backslash \Theta_{\text {low }}^{2 h} .
$$

To study how efficiently high-frequency error components are eliminated, smoothing factor $\mu$ is defined as

$$
\mu:=\sup _{\boldsymbol{\theta} \in \mathbf{\Theta}_{h i g h}^{2 h}} \rho\left(S_{h}(\boldsymbol{\theta})\right),
$$

where $S_{h}(\boldsymbol{\theta})$ represents the Fourier symbol of the relaxation operator. In our case, the Uzawa smoothing iteration can be written as $S_{h}=I_{h}-M_{h}^{-1} L_{h}$, where $L_{h}$ is the discrete operator given by the system matrix in (27) and $M_{h}$ represents the iteration matrix in (30).

In the transition from $G_{h}$ to $G_{2 h}$, each low frequency $\boldsymbol{\theta}=\boldsymbol{\theta}^{00} \in \boldsymbol{\Theta}_{\text {low }}^{2 h}$ is coupled with three high frequencies $\boldsymbol{\theta}^{11}, \boldsymbol{\theta}^{10}, \boldsymbol{\theta}^{01}$, given by

$$
\boldsymbol{\theta}^{i j}=\theta^{00}-\left(i \operatorname{sign}\left(\theta_{1}\right), j \operatorname{sign}\left(\theta_{2}\right)\right) \pi, \quad i, j=0,1 .
$$

For each $\theta^{00}$, three other Fourier modes $\varphi_{2 h}\left(\theta^{11}, \cdot\right), \varphi_{2 h}\left(\theta^{10}, \cdot\right)$, and $\varphi_{2 h}\left(\theta^{01}, \cdot\right)$ are identical to $\varphi_{2 h}\left(\theta^{00}, \cdot\right)$ on $G_{2 h}$. As a result, the Fourier space is subdivided into four-dimensional subspaces, known as $2 h$-harmonics,

$$
\mathcal{F}^{2 h}(\boldsymbol{\theta}):=\operatorname{span}\left\{\boldsymbol{\varphi}_{h}\left(\boldsymbol{\theta}^{00}, \cdot\right), \boldsymbol{\varphi}_{h}\left(\boldsymbol{\theta}^{11}, \cdot\right), \boldsymbol{\varphi}_{h}\left(\boldsymbol{\theta}^{10}, \cdot\right), \boldsymbol{\varphi}_{h}\left(\boldsymbol{\theta}^{01}, \cdot\right)\right\} \text {, with } \boldsymbol{\theta}=\boldsymbol{\theta}^{00} \in \boldsymbol{\Theta}_{\text {low }}^{2 h} \text {. }
$$

We can analyze the behavior of multigrid by investigating the effect of the multigrid components acting on the Fourier space. In particular, the iteration operator of the two-grid method is given by

$$
M_{h, 2 h}=S_{h}^{\nu_{2}}\left(I_{h}-P_{2 h, h}\left(L_{2 h}\right)^{-1} R_{h, 2 h} L_{h}\right) S_{h}^{\nu_{1}},
$$

where $\nu_{1}, \nu_{2}$ are the number of pre- and post-smoothing steps, $L_{h}$ and $L_{2 h}$ are the discrete operators on the two consecutive grids, $P_{2 h, h}$ and $R_{h, 2 h}$ are the prolongation and restriction operators, respectively, and $S_{h}$ is the relaxation, which in our case is the Uzawa smoothing iteration previously defined. Since the two-grid operator leaves the four-dimensional subspaces $\mathcal{F}^{2 h}(\boldsymbol{\theta})$ invariant, the representation of $M_{h, 2 h}$ on the Fourier space has a block-diagonal structure regarding the partitioning in $2 h$ harmonics [47], and therefore it is possible to efficiently calculate the LFA two-grid convergence factor,

$$
\rho=\rho\left(M_{h, 2 h}\right),
$$

by computing the maximum of the spectral radius of the blocks. 
LFA for the Uzawa smoother. A detailed study of the Uzawa smoother in the framework of LFA was already done in [23]. An analytic bound of the smoothing factor of the Uzawa smoother was given for a family of Stokes problems, showing a satisfactory approximation of the exact smoothing factor. In that paper, it was proved that

$$
\mu \leq \bar{\mu}=\max \left(\left(\mu_{A}\right)^{1 / 2}, \mu_{S}\right),
$$

where $\mu_{A}$ is the smoothing factor of $M_{A}$ and $\mu_{S}$ can be interpreted as the smoothing factor of the Richardson iteration for the Schur complement,

$$
\mu_{S}:=\sup _{\Theta_{\text {high }}^{2 h}} \rho\left(I-\omega\left(B A^{-1} B^{T}\right)\right) .
$$

There are no particular difficulties in obtaining bounds for $\mu_{A}$, since LFA results for many scalar elliptic PDEs are available in the literature; see, for example, [47]. However, to estimate $\mu_{S}$ is somewhat involved since information about the eigenvalues of the Schur complement is needed. In particular, the bound of $\mu_{S}$ is determined by the maximum and minimum eigenvalues on the high frequencies, that is,

$$
\begin{gathered}
\max _{\boldsymbol{\theta} \in \Theta_{h i g h}^{2 h}}\left(\widetilde{B}(\boldsymbol{\theta}) \widetilde{A^{-1}}(\boldsymbol{\theta}) \widetilde{B^{T}}(\boldsymbol{\theta})\right) \leq \beta_{\max }, \\
\min _{\boldsymbol{\theta} \in \Theta_{h i g h}^{2 h}}\left(\widetilde{B}(\boldsymbol{\theta}) \widetilde{A^{-1}}(\boldsymbol{\theta}) \widetilde{B^{T}}(\boldsymbol{\theta})\right) \geq \beta_{\min },
\end{gathered}
$$

with $\widetilde{B}(\boldsymbol{\theta}), \widetilde{A^{-1}}(\boldsymbol{\theta})$, and $\widetilde{B^{T}}(\boldsymbol{\theta})$ the symbols or Fourier representations of operators $B, A^{-1}$, and $B^{T}$ for a fixed frequency $\boldsymbol{\theta}$. Let $\zeta$ be a positive real number such that $\zeta<2$. By defining $\kappa_{\beta}=\frac{\beta_{\max }}{\beta_{\min }}$, the following bound for $\mu_{S}$ is obtained (see [23] for more details):

$$
\mu_{S} \leq \max \left(\zeta-1,1-\frac{\zeta}{\kappa_{\beta}}\right)
$$

Note that the choice of $\zeta<2$ is to ensure that $\mu_{S}<1$. Then, by choosing a value of $\zeta$ to minimize the expression in (44), we obtain an optimal relaxation parameter for the Uzawa smoother as follows:

$$
\omega=\frac{2}{\beta_{\max }+\beta_{\min }} .
$$

Next, we apply this analysis to obtain approximations of the smoothing factor of the Uzawa smoother for our problem, as well as optimal relaxation parameters for the Richardson iteration involved in the relaxation process.

In [23], the bound $\bar{\mu}=\max (0.5, \zeta-1)$ for the smoothing factor of the Uzawa smoother was obtained in the case of Stokes equations by choosing the optimal relaxation parameter $\omega=\zeta \nu$. Notice that $\mu_{A}=0.25$ for the symmetric Gauss-Seidel for the Laplace operator, and therefore $\left(\mu_{A}\right)^{1 / 2}=0.5$. These results can be directly used for our free-flow problem.

Uzawa smoother analysis for Darcy equation. We work out the analysis for Darcy's equation in order to obtain a suitable parameter $\omega$ for the part corresponding to the Richardson iteration for the pressure, as well as an approximation for the smoothing factor of the Uzawa smoother. 
Following the general analysis in the previous section to obtain $\beta_{\max }$ and $\beta_{\min }$ in (42)-(43), we will make use of the following equality:

$$
\widetilde{B}(\boldsymbol{\theta}) \widetilde{A^{-1}}(\boldsymbol{\theta}) \widetilde{B^{T}}(\boldsymbol{\theta})=K \widetilde{B}(\boldsymbol{\theta}) \widetilde{B^{T}}(\boldsymbol{\theta})=-K \widetilde{\Delta}(\boldsymbol{\theta}) .
$$

From this result, it is straightforward to obtain $\beta_{\max }=\frac{8 K}{h^{2}}$ and $\beta_{\min }=\frac{2 K}{h^{2}}$, which implies

$$
\kappa_{\beta}=\frac{\beta_{\max }}{\beta_{\min }}=4
$$

Choosing $\zeta=1.6$, which gives the lowest value of the maximum in (44), the smoothing factor is bounded by 0.6 , independently of the value of $K$. This theoretical bound for the smoothing factor $\bar{\mu}$ matches perfectly with the value $\mu$ predicted by the LFA. Moreover, following (45) the relaxation parameter is given by the expression $\omega=\frac{h^{2}}{5 K}$. Parameter $\omega$ depends on the grid size, and therefore it will be different on each grid of the hierarchy used in the multigrid method.

4.3. Multigrid for the coupled Darcy-Stokes problem. Due to the saddle point structure of the coupled problem, a geometric multigrid method together with an Uzawa smoother, as introduced in section 4.1, can be applied for the whole system. For this purpose, it is important to note that to keep the structure of the matrix of the saddle point system on the whole grid hierarchy, interface $\Gamma$ has to be present on each grid level. Regarding the smoothing process, all velocity unknowns are relaxed before the pressure unknowns are updated. The relaxation parameter $\omega$ for the Richardson iteration for the Schur complement has to be chosen differently if we are updating pressure unknowns from the Darcy or the Stokes problem. For the other components, the same operators can be used at every grid point since the discretization for both problems is performed with the same staggered arrangement of unknowns.

In the monolithic multigrid method we do not distinguish the subproblems and the internal interface. All the unknowns play essentially the same role. Only the relaxation parameter of the smoother is different for each subproblem. For the discretization at the interface, the unknowns for both subproblems are included in one equation. We keep the same philosophy for the other components in the monolithic multigrid. For example, to restrict the unknowns at the interface, six points from both subgrids around it are employed. A suitable discretization for the unknowns at the interface of the coupled system is a key step in achieving robustness and efficiency of our approach.

The proposed multigrid method for the coupled Darcy-Stokes problem can also be implemented as a multiblock version in which the Darcy and Stokes domains are assumed to be two different blocks. This is appealing from a practical point of view, for example, when one has to solve the coupled problem by using two different codes. Moreover, this multiblock approach is easily parallelizable. Next, we describe in detail how this implementation can be done.

Multiblock multigrid algorithm. We divide our domain into two different blocks corresponding to the Darcy and Stokes domains. In this way, the original staggered grid is split into two different subgrids. Starting with an approximation on the partitioned grid, it is trivial to compute a new iterate for the interior points of each subgrid. Near the boundary of each subgrid, the old approximations at those points belonging to the neighboring subgrid are needed. It is standard within a gridpartitioning framework [45] for a subgrid to store not only its own data but also a 
copy of the data located in a strip of the neighboring subgrid in an overlap region. Thus, the mesh corresponding to the Stokes domain is extended by adding an overlap region of one cell length, as can be seen in Figure 5. After a full iteration, on each grid level, the copies in the overlap region have to be updated by communication so that a next iteration can be carried out.
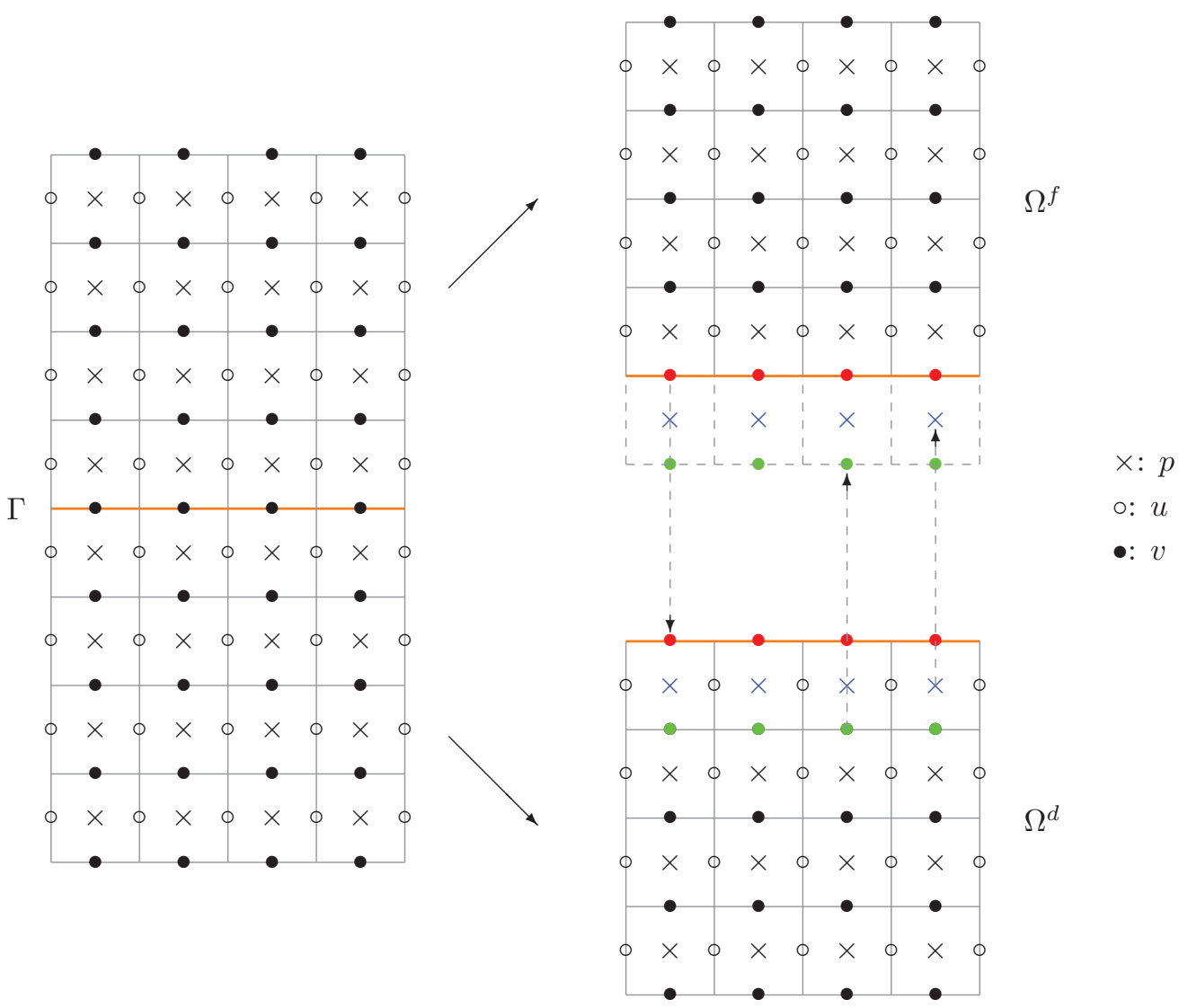

FIG. 5. Communications between two partitioned subgrids.

Next, we explain in detail the two-grid version of the multiblock algorithm. For simplicity in the presentation of the algorithm, we use pre-smoothing but no postsmoothing. By recursion, the multigrid version follows straightforwardly.

Multiblock two-grid algorithm (with pre-smoothing but no post-smoothing):

1. Relax velocity unknowns for both blocks.

2. Stokes to Darcy transfer: vertical Stokes velocity unknowns at the interface are transferred to the Darcy block (see the red dots in Figure 5).

3. Update pressure unknowns by the Richardson iteration with the optimal relaxation parameters corresponding to each block.

4. Darcy to Stokes transfer: Darcy pressure unknowns are transferred to the Stokes overlap region (see the blue crosses in Figure 5).

5. Compute the residual.

Copyright $@$ by SIAM. Unauthorized reproduction of this article is prohibited. 
6. Darcy to Stokes transfer: the residual of the vertical Darcy velocity unknowns is transferred to the Stokes overlap region (see the green dots in Figure 5).

7. Restrict the residual.

8. Solve exactly the defect equation on the coarsest grid.

9. Stokes to Darcy transfer: vertical Stokes velocity unknowns at the interface are transferred to the Darcy block.

10. Interpolate the error and correct the approximation to the solution.

This multiblock algorithm requires little data communication. In particular, each communication step involves transfer of information in only one way. Moreover, each stage in the algorithm can be performed in parallel since the data required for each operation is available in the same process. Finally, although this multiblock approach can be cast into the class of DDMs, we wish to emphasize that in our case the communication between both Darcy and Stokes problems is performed on each level in the hierarchy instead of only on the finest grid as is usually done in the DDMs. This is crucial to achieve a highly efficient solver for this coupled problem, as we will see in the numerical experiments section.

LFA results. In this section, we confirm that the asymptotic convergence factor of the monolithic multigrid based on the Uzawa smoother for the coupled problem can be estimated with a high accuracy by means of the worst of the two-grid convergence factors predicted by LFA for the individual Darcy and Stokes subproblems. In Table 1, we display the two-grid convergence factors predicted by the LFA for the Darcy problem varying the hydraulic conductivity $K$, and for the Stokes equations for different values of the viscosity $\nu$. These results are obtained for different numbers of smoothing steps $\nu_{1}+\nu_{2}$. From this table, we can observe the robustness of the multigrid method based on the Uzawa smoother for each subproblem, separately.

TABLE 1

Two-grid convergence factors, $\rho$ predicted by LFA for Darcy and Stokes subproblems, separately, for different values of the parameters $K$ and $\nu$ and different numbers of smoothing steps $\nu_{1}+\nu_{2}$.

\begin{tabular}{|c|c|c|c|c|c|c|}
\cline { 2 - 7 } \multicolumn{1}{c|}{} & \multicolumn{3}{c|}{ Darcy } & \multicolumn{3}{c|}{ Stokes } \\
\hline$\nu_{1}+\nu_{2}$ & $K=1$ & $K=10^{-3}$ & $K=10^{-6}$ & $\nu=1$ & $\nu=10^{-3}$ & $\nu=10^{-6}$ \\
\hline 2 & 0.600 & 0.600 & 0.600 & 0.304 & 0.304 & 0.304 \\
3 & 0.360 & 0.360 & 0.360 & 0.143 & 0.143 & 0.143 \\
4 & 0.216 & 0.216 & 0.216 & 0.081 & 0.081 & 0.081 \\
\hline
\end{tabular}

TABLE 2

Asymptotic convergence factors, $\rho_{h}$, for the multigrid based on Uzawa smoother for the coupled Darcy-Stokes problem, for different values of the physical parameters $K$ and $\nu$ and different numbers of smoothing steps $\nu_{1}+\nu_{2}$.

\begin{tabular}{|c|c|c|c|c|c|c|c|c|c|c|}
\cline { 2 - 11 } \multicolumn{1}{c|}{} & $K$ & \multicolumn{3}{c|}{1} & \multicolumn{3}{c|}{$10^{-3}$} & \multicolumn{3}{c|}{$10^{-6}$} \\
\cline { 2 - 11 } \multicolumn{1}{c|}{} & $\nu$ & 1 & $10^{-3}$ & $10^{-6}$ & 1 & $10^{-3}$ & $10^{-6}$ & 1 & $10^{-3}$ & $10^{-6}$ \\
\hline \multirow{3}{*}{$\nu_{1}+\nu_{2}$} & 2 & 0.59 & 0.59 & 0.59 & 0.59 & 0.59 & 0.59 & 0.59 & 0.59 & 0.59 \\
& 3 & 0.36 & 0.36 & 0.36 & 0.36 & 0.36 & 0.36 & 0.36 & 0.36 & 0.36 \\
& 4 & 0.21 & 0.21 & 0.21 & 0.21 & 0.21 & 0.21 & 0.21 & 0.21 & 0.21 \\
\hline
\end{tabular}

In Table 2, we show the asymptotic convergence factors experimentally obtained by using the monolithic multigrid method based on the Uzawa smoother for the Darcy-Stokes coupled problem. These values have been computed on a fine grid of 
size $h=1 / 128$ and by using a random initial guess and zero right-hand side in order to avoid round-off errors. We display the convergence factors obtained after 100 multigrid cycles, since in each test case the numerical convergence has stabilized. Comparing Tables 1 and 2, we observe that these factors match perfectly with the worst of the twogrid convergence factors predicted by LFA for both separate subproblems. This means that the treatment of the discretization at the interface as well as the implementation of the Uzawa smoother for the whole coupled problem have been performed in the most efficient way.

Discussion and comparison with other methods. In this section we discuss alternative solution methods to the proposed monolithic multigrid approach. In the discussion we include, on the one hand, monolithic multigrid methods with different smoothers, and on the other hand, domain decomposition techniques and preconditioning strategies.

In a monolithic multigrid method we aim to solve the multiphysics system all at once. The choice of the smoother is important for the performance of the multigrid method. The proposed smoother in our work is based on Uzawa relaxation, but different relaxation schemes can be considered. The Braess-Sarazin method $[1,5]$ is an example of another relaxation method. More concretely, the Braess-Sarazin method is based on the matrix system

$$
\left(\begin{array}{cc}
N_{A} & B^{T} \\
B & 0
\end{array}\right)
$$

as the smoothing iteration matrix for saddle point system (27), where $N_{A}$ is usually of the form $N_{A}:=\alpha \operatorname{diag}(A)$ or $N_{A}:=\alpha I$, with $\alpha \in \mathbb{R}$ a parameter which is not smaller than the maximum eigenvalue of $A$. For the solution of the pressure it is then necessary to solve a system whose matrix involves the Schur complement $B N_{A}^{-1} B^{T}$. However, in practice, an inexact solve is sufficient. In that case, an inexact Braess-Sarazin smoother is comparable to the Uzawa iteration and seems an appealing alternative to the Uzawa smoother. Next to the Braess-Sarazin smoother, the well-known coupled Vanka smoother [46] is based on solving several small-sized and local saddle point problems in a block Gauss-Seidel fashion. Originally proposed for the incompressible Navier-Stokes equations, this approach can be easily extended to solving the Darcy-Stokes system. The interface conditions, discretized on a staggered grid, can be naturally incorporated within the coupled Vanka smoothing approach. In particular, in the staggered case that we consider, five unknowns (pressure $p_{i, j}$ and velocities $u_{i-\frac{1}{2}, j}, u_{i+\frac{1}{2}, j}, v_{i, j-\frac{1}{2}}$, and $v_{i, j+\frac{1}{2}}$ ) are simultaneously updated (see Figure 3 ), which results in solving $5 \times 5$ systems for each cell in the grid. A coupled smoother is often somewhat more expensive than an equationwise smoother. We will compare the Uzawa smoother with the Vanka smoother, in terms of computational efficiency, in the numerical section. Note, however, that the performance of a pointwise coupled smoother is not satisfactory when stretched grids or anisotropic problem parameters are encountered (see [22], for example), and their extension to linewise Vanka relaxation gives rise to a significant increase in computational cost, since several lines of unknowns have to be updated simultaneously.

As mentioned in the introduction, other approaches for solving coupled problems are based on DDMs, splitting the multiphysics system into separate subproblems that are treated mainly independently, as specific preconditioning techniques for the global saddle point problem. In this context, preconditioned GMRES methods with block or constraint preconditioners $[10,15,16]$ usually show a mesh-independent convergence 
rate, yielding an effective approach for solving the Darcy-Stokes problem. However, in the literature it can be observed that these methods can exhibit some parameter specific convergence, depending on the values of the physical parameters (see [11], for example). Our monolithic method provides convergence characteristics independent of parameters $K$ and $\nu$. Even for very small parameter values, when the subproblems are strongly coupled, the multigrid convergence is excellent. One of the advantages of the considered monolithic multigrid method is thus the robustness with respect to the values of the physical parameters.

Remark. As is commonly done, in this work, we mainly focus on the case of constant hydraulic conductivity $K$. The proposed multigrid solution method can, however, be generalized to varying $K$ values and also to the case where the hydraulic conductivity is prescribed by a full tensor $\mathbb{K}$. In [34], we applied a variant of the Uzawa smoother for porous media flow when anisotropies due to grid stretching appeared. Also, heterogeneous coefficients were considered in that work. Grid anisotropies basically have the same impact as anisotropic conductivity. Therefore, the proposed algorithm can be adapted to such a setting. When a full SPD tensor $\mathbb{K}$ is encountered, the same solution strategy may be applied since $\mathbb{K}$ can be diagonalized. For the case of a heterogeneous porous medium, some multigrid results are presented in Appendix A.

5. Numerical experiments. We present three numerical tests in order to study the accuracy of the discrete scheme and the convergence and robustness of the proposed multigrid method based on the Uzawa smoother with respect to different values of the kinematic viscosity $\nu$ and the hydraulic conductivity $K$. For the implementation, we will consider the optimal relaxation parameters for the Richardson iteration defined in section 4 , with values of $\zeta=1$ for the Stokes problem and $\zeta=1.6$ for the Darcy problem. For Stokes it follows that $\omega=\nu$, that is, the relaxation parameter is fixed on all grids and equal to the viscosity of the fluid. This is due to the fact that the Schur complement is spectrally equivalent to the identity matrix for the considered discretization, and therefore the eigenvalues are bounded from below and above by positive constants which do not depend on the mesh size. $\omega=\frac{h^{2}}{5 K}$ in the Darcy domain, so $\omega$ depends on $K$, which is the hydraulic conductivity of the porous media, and on the size of the grid (different on each mesh in the hierarchy).

In all numerical experiments, the initial solution is chosen to be random numbers, and the stopping criterion is

$$
\frac{\| \text { residual } \|_{\infty}}{\| \text { right-hand side } \|_{\infty}} \leq \text { tolerance } \cdot \frac{\| \text { initial residual } \|_{\infty}}{\| \text { right-hand side } \|_{\infty}}
$$

where the tolerance is chosen as $10^{-10}$. Moreover, for the sake of simplicity we consider uniform meshes with grid size $h$ in both directions on each subdomain.

5.1. No-slip interface condition. In this first numerical experiment we deal with a coupled Darcy-Stokes problem with a known analytic solution on the domain $\Omega=(0,1) \times(0,2)$, which is a benchmark test widely used to assess the behavior of different numerical algorithms; see [11, 19, 20], for example. The domain $\Omega$ is divided into two subdomains by the interface $\Gamma=(0,1) \times\{1\}$. The Stokes region is the upper part $\Omega^{f}=(0,1) \times(1,2)$, whereas the Darcy region is the bottom part $\Omega^{d}=(0,1) \times(0,1)$. We choose the right-hand side terms and the boundary conditions 
so that the exact solution is given by

$$
\begin{aligned}
& \mathbf{u}^{f}(x, y)=\left(\begin{array}{c}
u^{f}(x, y) \\
v^{f}(x, y)
\end{array}\right)=\left(\begin{array}{c}
y^{2}-2 y+1 \\
x^{2}-x
\end{array}\right), \\
& p^{f}(x, y)=2 \nu(x+y-1)+\frac{1}{3 K}, \\
& \mathbf{u}^{d}(x, y)=\left(\begin{array}{c}
u^{d}(x, y) \\
v^{d}(x, y)
\end{array}\right)=\left(\begin{array}{c}
(2 x-1)(y-1)-2 K \nu \\
\left(x^{2}-x\right)-y^{2}+2 y-1
\end{array}\right), \\
& p^{d}(x, y)=\frac{1}{K}\left(\left(x-x^{2}\right)(y-1)+\frac{y^{3}}{3}-y^{2}+y\right)+2 \nu x .
\end{aligned}
$$

Dirichlet boundary conditions for velocity are prescribed at $\partial \Omega^{f} \backslash \Gamma$ and at the bottom boundary $(0,1) \times\{0\}$. Neumann boundary conditions for pressure are imposed at the remaining parts, i.e., the lateral boundaries of the porous medium. Moreover, a simplified no-slip interface condition (13), together with (10) and (11), is considered here at the internal interface.

First of all, we compare the numerical solution with the given exact solution for fixed values of viscosity $\nu=1$ and hydraulic conductivity $K=1$. For different grid sizes, in Table 3 we display the maximum norm of the error for each variable. As expected, second-order accuracy is obtained for the Darcy problem, whereas for the Stokes problem, we achieve second-order accuracy for velocities and first-order accuracy for the pressure field. The maximum errors for vertical velocities are the same in both subdomains due to the fact that such a maximum is achieved at the internal interface $\Gamma$.

TABLE 3
Maximum norm errors of variables $u^{d / f}, v^{d / f}, p^{d / f}$ for different grid sizes, by considering fixed values $\nu=1$ and $K=1$.

\begin{tabular}{|c|c|c|c|c|}
\hline & $32 \times 64$ & $64 \times 128$ & $128 \times 256$ & $256 \times 512$ \\
\hline$u^{d}$ & $2.03 \times 10^{-4}$ & $5.38 \times 10^{-5}$ & $1.39 \times 10^{-5}$ & $3.51 \times 10^{-6}$ \\
$v^{d}$ & $3.11 \times 10^{-4}$ & $8.81 \times 10^{-5}$ & $2.34 \times 10^{-5}$ & $6.02 \times 10^{-6}$ \\
$p^{d}$ & $2.43 \times 10^{-4}$ & $6.09 \times 10^{-5}$ & $1.52 \times 10^{-5}$ & $3.81 \times 10^{-6}$ \\
\hline$u^{f}$ & $2.29 \times 10^{-4}$ & $5.91 \times 10^{-5}$ & $1.50 \times 10^{-5}$ & $3.78 \times 10^{-6}$ \\
$v^{f}$ & $3.11 \times 10^{-4}$ & $8.81 \times 10^{-5}$ & $2.34 \times 10^{-5}$ & $6.02 \times 10^{-6}$ \\
$p^{f}$ & $3.61 \times 10^{-2}$ & $1.81 \times 10^{-2}$ & $9.07 \times 10^{-3}$ & $4.54 \times 10^{-3}$ \\
\hline
\end{tabular}

Now we focus on the study of the behavior of the proposed multigrid method for the Darcy-Stokes problem. First, a multigrid $W$-cycle with two pre- and two post-smoothing steps is applied in order to see the $h$-independent convergence of the algorithm for fixed values $\nu=K=1$. In Figure 6 we show the history of the convergence for different grid sizes $h=1 / 2^{k}$ for $k=5,6,7,8$. The maximum norm of the residuals divided by the maximum norm of the right-hand sides is plotted in logarithmic scale against the number of multigrid cycles necessary to fulfill the stopping criterion. It can be seen that the convergence rate is independent of the space discretization parameter, and that the proposed multigrid method performs well for the coupled problem, since only 15 iterations are needed to achieve the desired convergence.

Next, we investigate the robustness of the multigrid algorithm with respect to a wide range of values of the physical parameters $\nu$ and $K$. This is important since the 


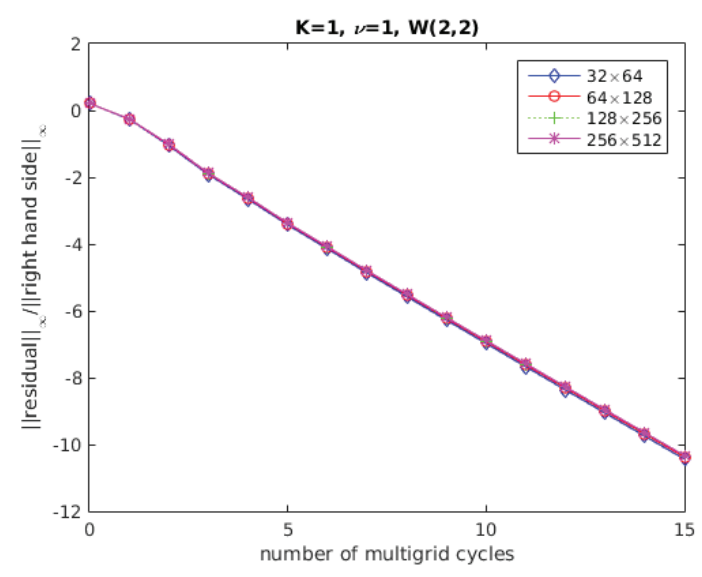

FIG. 6. History of the convergence of the $W(2,2)$-multigrid method for different grids.

values of the parameters which are relevant for geoscientific applications are typically very small. For example, $K=10^{-2}$ is the hydraulic conductivity for sand and $K=$ $10^{-5}$ for limestone, and $\nu=10^{-6}$ is the viscosity of water. In Table 4 , we show the number of iterations necessary to reach the stopping criterion for different values of $\nu$ and $K$, and for different multigrid cycles and numbers of pre- and post-smoothing steps. All of these results are obtained on a grid with space discretization parameter $h=\frac{1}{128}$.

TABLE 4

Number of iterations necessary to achieve the desired convergence for different values of the parameters $\nu$ and $K$, by using $W$ - and $V$-cycles with different numbers of pre- and post-smoothing steps.

\begin{tabular}{|c|c||c|c|c||c|c|}
\hline$K$ & $\nu$ & $W(1,1)$ & $W(1,2)$ & $W(2,2)$ & $V(2,2)$ & $V(3,3)$ \\
\hline 1 & 1 & 38 & 21 & 15 & 22 & 13 \\
\hline $10^{-3}$ & 1 & 38 & 20 & 14 & 28 & 13 \\
\hline 1 & $10^{-3}$ & 45 & 24 & 17 & 32 & 14 \\
\hline $10^{-3}$ & $10^{-3}$ & 38 & 20 & 14 & 23 & 10 \\
\hline $10^{-2}$ & $10^{-6}$ & 39 & 21 & 15 & 24 & 11 \\
\hline $10^{-4}$ & $10^{-6}$ & 37 & 20 & 14 & 22 & 9 \\
\hline $10^{-6}$ & $10^{-6}$ & 37 & 20 & 14 & 22 & 9 \\
\hline $10^{-7}$ & $10^{-6}$ & 37 & 20 & 14 & 22 & 9 \\
\hline
\end{tabular}

As can be observed, the proposed multigrid method results in a robust solver when $W$-cycles are used. If $V$-cycles are chosen, we can also observe a satisfactory behavior of the multigrid method. Moreover, very good results are obtained for small values of the physical parameters. In order to analyze the efficiency of the proposed method, we have done a comparison of the number of arithmetic operations needed for both cycles. The most efficient multigrid cycle in Table 4 is the $V(3,3)$-cycle. Therefore, we choose this cycle to compare the efficiency of the proposed multigrid based on the Uzawa smoother with a multigrid algorithm based on the Vanka smoother. For these two methods, the only difference is the smoothing part on each level of the 
hierarchy. By calculating the computational work per $V$-cycle, we observe that the Uzawa smoother is approximately $30 \%$ cheaper than the Vanka smoother. In Table 5, the number of multigrid cycles necessary to achieve the desired accuracy is presented. Overall, the method based on the Uzawa relaxation needs 50\% fewer operations than the Vanka-based multigrid method. Therefore, in our case the Uzawa smoother is preferred to the Vanka relaxation.

TABLE 5

Number of iterations necessary to achieve the desired convergence for different values of the parameters $\nu$ and $K$, by using a $V(3,3)$-cycle with both Uzawa and Vanka smoothers.

\begin{tabular}{|c||c|c|c|c|c|c|c|c|}
\hline$K$ & 1 & $10^{-3}$ & 1 & $10^{-3}$ & $10^{-2}$ & $10^{-4}$ & $10^{-6}$ & $10^{-7}$ \\
\hline$\nu$ & 1 & 1 & $10^{-3}$ & $10^{-3}$ & $10^{-6}$ & $10^{-6}$ & $10^{-6}$ & $10^{-6}$ \\
\hline Uzawa & 13 & 13 & 14 & 10 & 11 & 9 & 9 & 9 \\
\hline Vanka & 12 & 11 & 13 & 11 & 12 & 11 & 11 & 11 \\
\hline
\end{tabular}

5.2. Beavers-Joseph-Saffman interface condition. Now we consider a more complicated and realistic numerical test in which the Beavers-Joseph-Saffman interface condition is prescribed instead of the no-slip condition previously considered. In this case, the domain $\Omega=(0,1) \times(-1,1)$ is divided into a porous medium part $\Omega^{d}=(0,1) \times(-1,0)$ and a free-flow subdomain $\Omega^{f}=(0,1) \times(0,1)$ by the interface $\Gamma=(0,1) \times\{0\}$. The source terms and the boundary conditions are chosen such that the analytic solution of the Darcy-Stokes problem is as follows:

$$
\begin{aligned}
& \mathbf{u}^{d}(x, y)=\left(\begin{array}{c}
u^{d}(x, y) \\
v^{d}(x, y)
\end{array}\right)=\left(\begin{array}{c}
-K e^{y} \cos x \\
-K e^{y} \sin x
\end{array}\right), \\
& p^{d}(x, y)=e^{y} \sin x, \\
& \mathbf{u}^{f}(x, y)=\left(\begin{array}{c}
u^{f}(x, y) \\
v^{f}(x, y)
\end{array}\right)=\left(\begin{array}{c}
\lambda^{\prime}(y) \cos x \\
\lambda(y) \sin x
\end{array}\right), \\
& p^{f}(x, y)=0
\end{aligned}
$$

where $\lambda(y)=-K-\frac{y}{2 \nu}+\left(-\frac{\alpha}{4 \nu^{2}}+\frac{K}{2}\right) y^{2}$. At the outer boundaries of the free-flow domain, Dirichlet boundary conditions for velocities are prescribed. In the case of the porous medium, the pressure is fixed at the bottom, $(0,1) \times\{-1\}$, whereas Dirichlet conditions for velocities are imposed at the lateral walls. Along the internal interface $\Gamma$, the Beavers--Joseph-Saffman condition (12) is taken into account.

By comparing the numerical solution with the given exact solution for fixed values of the parameters $\nu=K=1$ and for different grid sizes $h=1 / 2^{k}$ for $k=5,6,7,8$, second-order accuracy is again obtained for all variables except for the pressure in the free-flow subdomain, where we achieve first-order accuracy. This time the errors for the vertical velocities do not reach their maximum at the interface as in the previous numerical test in which we imposed the no-slip condition instead the Beavers-JosephSaffman condition.

Regarding the performance of the monolithic multigrid method for the coupled problem considered in this numerical test, we display in Figure 7 the history of the convergence of the algorithm by using a $W(2,2)$-cycle for different grids and $\nu=K=$ 1. The $W(2,2)$-cycle is chosen here since it gives a more robust multigrid method. It is clear that the convergence is independent of the mesh size and that the method performs efficiently since it needs only around 13 iterations to achieve the required 
stopping criterion. In Figure 8 the robustness of the proposed multigrid method is displayed, since for different values of $\nu$ and $K$ and different grid sizes the convergence of the algorithm is highly satisfactory and independent of the parameters. We can observe that with the more complicated Beavers-Joseph-Saffman condition at the interface $\Gamma$, the results provided by the proposed multigrid method for the DarcyStokes problem are highly satisfactory.

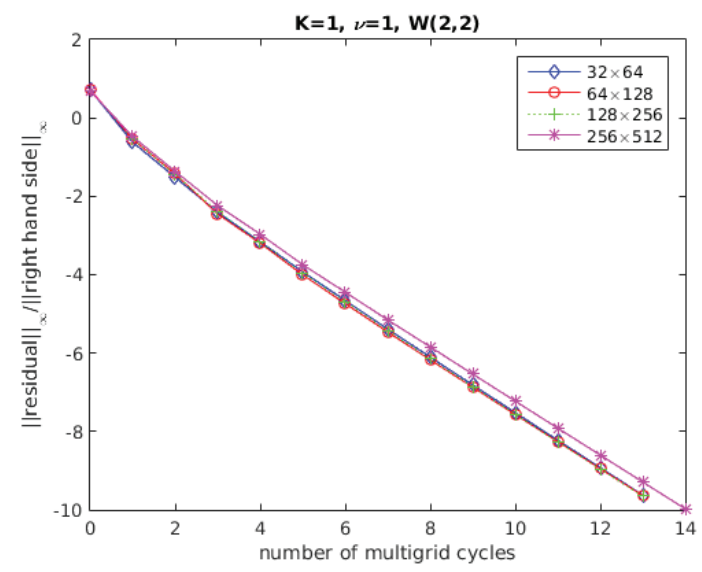

FIG. 7. History of the convergence of the W(2,2)-multigrid method when the Beavers-JosephSaffman interface condition is considered.

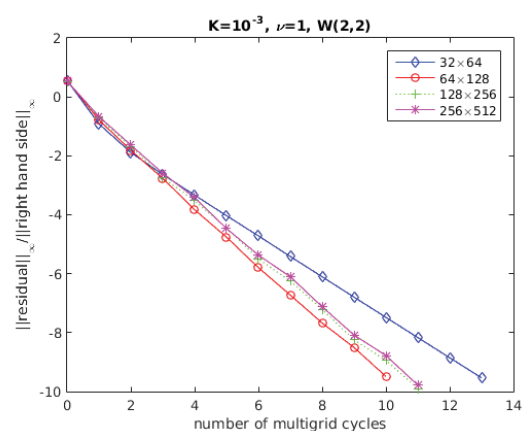

(a)

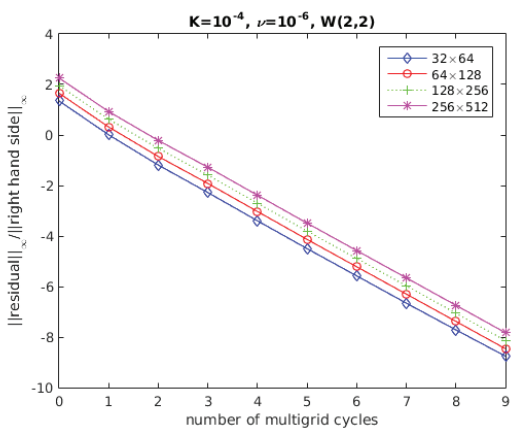

(b)

FIG. 8. History of the convergence of the $W(2,2)$-multigrid method when the Beavers-JosephSaffman interface condition is considered for different values of the physical parameters: (a) $\nu=1$, $K=10^{-3}$, and (b) $\nu=10^{-6}, K=10^{-4}$.

5.3. Realistic test: Cross-flow membrane filtration model. This test addresses the coupling of the Darcy-Stokes problem which is in a cross-flow filtration setting. The cross-flow filtration can be applied in a wide range of industrial applications ranging from oil production to medical treatment. The data in this test is taken from the experiment presented in [24], which is a micromembrane filtration model. This model is used to clean fluids that are difficult to filter and to separate fine matter such as cells, proteins, enzymes, and viruses [24].

The domain of the coupled problem is shown in Figure 9. $\Omega^{f}$ represents a channel 


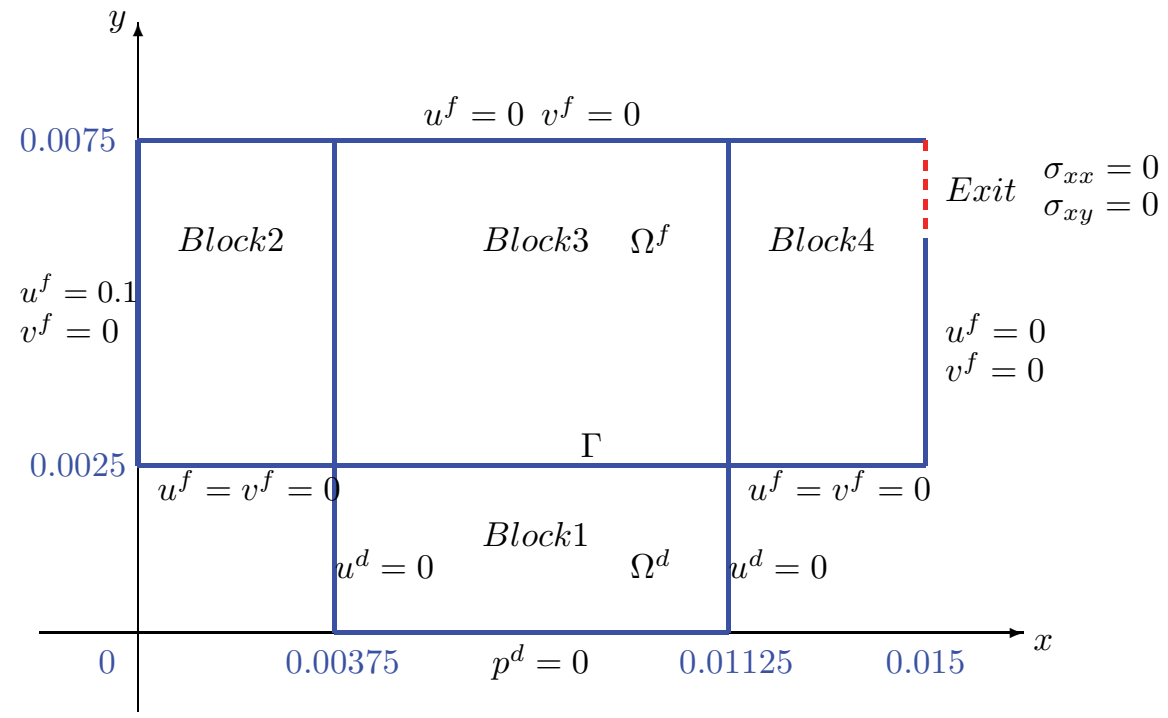

FIG. 9. Geometry of the cross-flow membrane filtration model. Subdivision of the domain $\Omega$ into a free-flow subregion $\Omega^{f}$ and a filter subdomain $\Omega^{d}$, by an internal interface $\Gamma$.

on the top where the flow can go through, while $\Omega^{d}$ represents a filter. Since the lengths of the free-flow domain and the porous medium are not the same, the coupled domain is divided into four different blocks corresponding to the Darcy (Block1) and Stokes (Block2, Block3, and Block4) domains. The two-block multigrid algorithm described in section 4.3 can be straightforwardly adapted for fours blocks. The information transfer between Block 1 and Block 3 is the same as before. For the Stokes domain, two artificial boundaries are generated by the partitioning. As the communication between the subgrids in $\Omega^{f}$ is necessary, an overlap region of one cell length is created for Block 2 and Block 4 along the artificial boundaries. The data located in the overlap region is computed and transferred from the neighboring subgrid in Block3.

The unknowns at the artificial boundaries, i.e., $u^{f}$, are updated in Block 2 and Block4, and then sent to Block3. The communication is implemented on each level in the multigrid algorithm. The inflow entering into the domain $\Omega^{f}$ is specified. At the interface, the Beavers-Joseph-Saffman condition is imposed. At the bottom of the porous medium, the pore pressure is set as zero. There is an exit (see the dashed line in Figure 9) at the right vertical boundary of the free-flow domain. The height of the exit is 0.00125 , which is quite small compared to the inlet. The stress-free boundary condition is employed at the exit, where the flow may leave the domain freely. All the other imposed conditions are shown in Figure 9.

Two values of hydraulic conductivity, $K=0.1$ and $K=10^{-6}$, are considered in the numerical experiment. The fluid viscosity is chosen as $10^{-6}$. The solutions are investigated on four grids, as shown in Table 6 . For the test with $K=0.1$, the velocity components along the vertical and horizontal centerlines are shown in Figure 10. It can be seen that the solutions for Grid3 and Grid4 do not differ much. This indicates that the numerical solution is convergent with the increase of the grid cells, and the exact solution is closely approximated on these grids. For a multigrid $W(2,2)$-cycle, the multigrid convergence factor is around 0.2 for all cases, and the multigrid method exhibits a highly satisfactory behavior. This is in accordance with the previous tests. 
TABLE 6

Different grids in the computational tests.

\begin{tabular}{|l|c|c|c|c|}
\hline \hline & Block1 & Block2 & Block3 & Block4 \\
\hline Grid1 & $192 \times 64$ & $96 \times 128$ & $192 \times 128$ & $96 \times 128$ \\
Grid 2 & $96 \times 32$ & $48 \times 64$ & $96 \times 64$ & $48 \times 64$ \\
Grid3 & $48 \times 16$ & $24 \times 32$ & $48 \times 32$ & $24 \times 32$ \\
Grid4 & $24 \times 8$ & $12 \times 16$ & $24 \times 16$ & $12 \times 16$ \\
\hline \hline
\end{tabular}

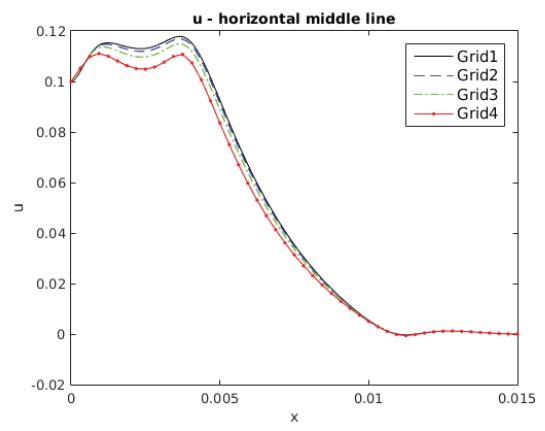

(a) $x=0.0075$.

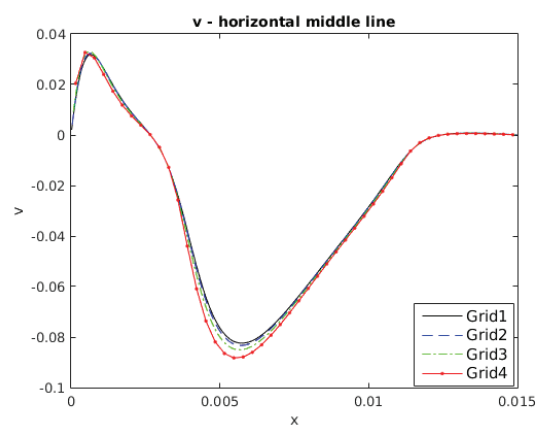

(c) $x=0.0075$.

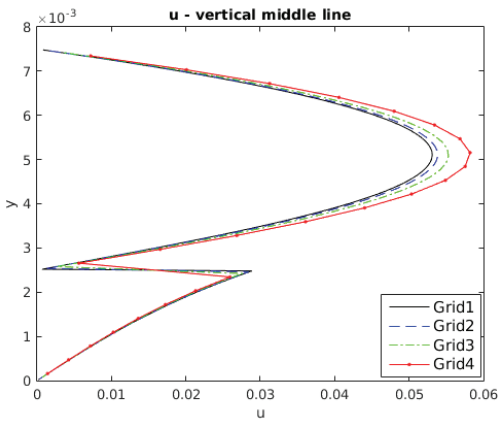

(b) $y=0.00375$.

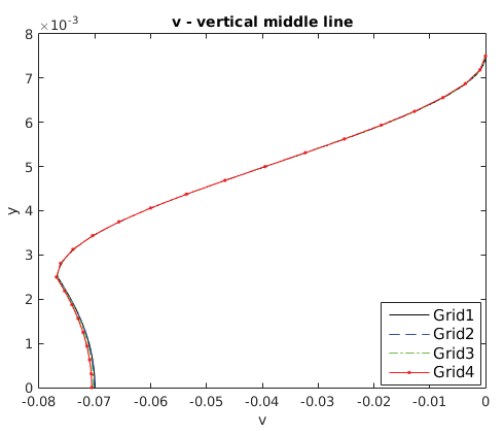

(d) $y=0.00375$.

FIG. 10. Velocity component (a) $u$ along the vertical centerline in the coupled domain, (b) $u$ along the horizontal centerline in the coupled domain, (c) $v$ along the vertical centerline in the coupled domain, and (d) $v$ along the horizontal centerline in the coupled domain.

In Figure 11, we show the velocity vector corresponding to $K=0.1$. Since the hydraulic conductivity of the porous medium is quite high, when the fluid travels tangentially across the interface, the majority of the flow seeps into the filter, while only a small amount of fluid goes through the exit of the channel.

In Figure 12, the velocity vector corresponding to $K=10^{-6}$ is represented. With such a low hydraulic conductivity of the porous medium, the minority of the flow penetrates the interface, whereas most of the fluid flows toward the small exit of the channel. 


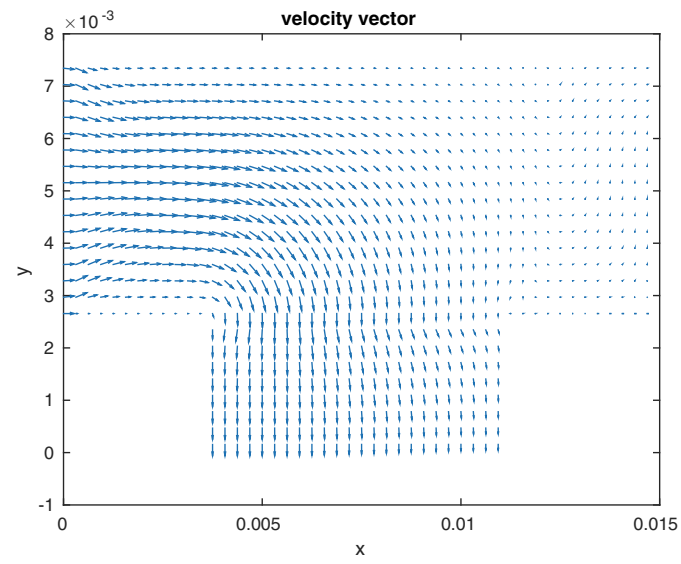

FIG. 11. Velocity vectors over the cross-flow filtration domain with hydraulic conductivity $K=0.1$.

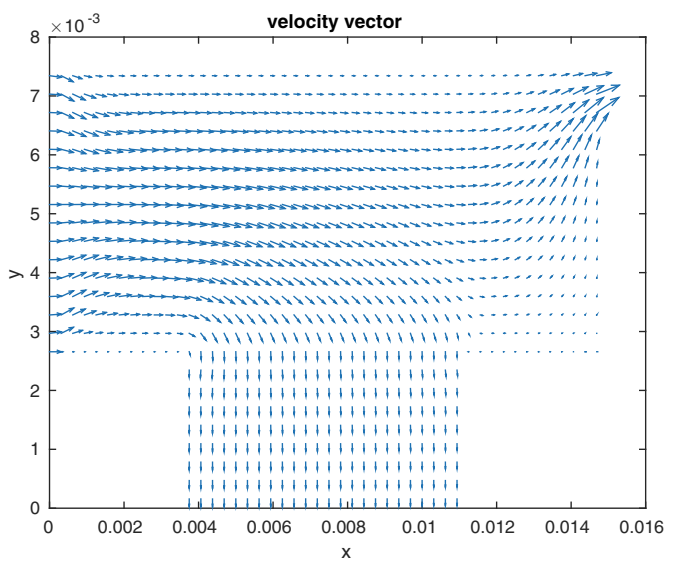

FIG. 12. Velocity vectors over the cross-flow filtration domain with hydraulic conductivity $K=$ $10^{-6}$. For easier viewing, the vectors have been scaled by 1.5 .

6. Conclusions. In this paper, we investigated the multigrid convergence of a coupled system consisting of a porous medium and incompressible flow. For this purpose, we formulated a coupled model based on the Darcy equation and the incompressible Stokes equations with appropriate internal interface conditions. The model is discretized by finite volumes on a staggered grid, and special care has been taken regarding the accurate discretization at the interface. We focused on an efficient multigrid algorithm for the coupled problem. A decoupled Uzawa smoother is employed, which is based on symmetric Gauss-Seidel smoothing for the velocity components, and a simple Richardson iteration on the Schur complement to update the pressure field. By local Fourier analysis we have selected suitable relaxation parameters for both systems, and we have confirmed the global convergence of the monolithic multigrid, which proves to be the worst of the convergence factors of the individual Darcy and Stokes subproblems. Numerical tests have shown a highly satisfactory convergence of our multigrid method for the coupled system. The algorithm performed very well in numerical experiments for a wide range of physical parameter values and for 
different interface conditions.

Appendix A. Heterogeneity test. Often, a porous medium is defined by complicated material properties. Therefore, here we consider a porous medium with a random heterogeneous hydraulic conductivity $K$. Our aim is to study the effect of this heterogeneity model on the multigrid convergence. To simulate heterogeneity in the porous medium, a statistical approach is chosen. In order to generate random spatial data, a Gaussian model characterized by parameters $\lambda_{g}$ and $\sigma_{g}^{2}$ is considered, i.e.,

$$
C\left(d_{g}\right)=\sigma_{g}^{2} \exp \left(-\frac{d_{g}^{2}}{\lambda_{g}}\right),
$$

where $d_{g}$ is the distance between two points, $\lambda_{g}$ defines the correlation length, and $\sigma_{g}^{2}$ represents the variance. By using a so-called circulant embedding technique, outlined in [43], we generate a random field on a vertex-centered grid which is twice as fine as the computational grid. As an example, in Figure 13 we present a possible random sample of the hydraulic conductivity $K$ corresponding to the porous medium in Figure 9 with parameters $\lambda_{g}=0.3$ and $\sigma_{g}^{2}=1$. Dark blue in Figure 13 represents a higher value of the hydraulic conductivity, whereas dark red is for low conductivity.

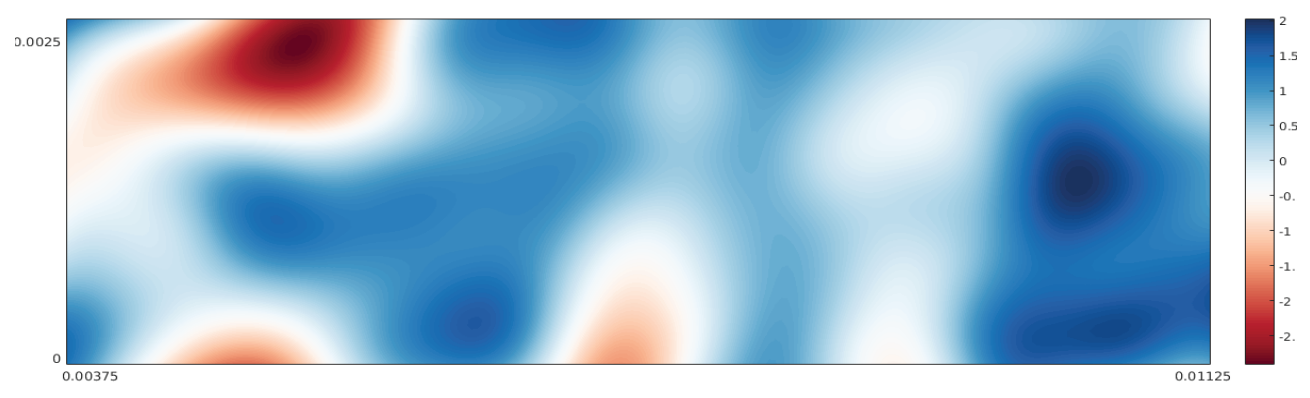

FIG. 13. Example of random field of hydraulic conductivity $K$ in log-scale.

Note that when our multigrid algorithm with Uzawa smoother is applied, the relaxation parameter $\omega$ is varied in the Darcy domain, because $\omega$ depends on hydraulic coefficient $K$. The corresponding suitable relaxation parameters for each grid point on each grid level can be calculated and used within the Uzawa smoother. Moreover, the random field should be transferred from the finest grid to the other grid levels, to guarantee the same characteristics of the porous medium on fine and coarse grids.

In our current experiment, for solving the problem in section 5.3, two different values for parameter $\lambda_{g}$ are chosen to analyze the multigrid convergence results; $\lambda_{g}=$ 0.1 denotes a more heterogeneous porous medium than $\lambda_{g}=0.3$. Solutions are computed on three grids, as indicated in Table 6 . Conductivity $K$ is restricted to each grid level as the average value of four neighboring fine grid points [30]. For each case, 50 realizations of the random field are generated, and we record the multigrid convergence factors of the $W(2,2)$-cycle. The mean value of the convergence factors is presented in Table 7 . Since fine grids are able to represent the field more accurately, the convergence results are improved with grid refinement. Multigrid exhibits better convergence for the less heterogeneous porous medium. In Figure 14, the solutions for $\lambda_{g}=0.3$ are depicted. As expected, the velocity in the porous medium is higher 
(blue and white in Figure 14) where the value of hydraulic conductivity is higher (Figure 13).

TABLE 7

Mean value of the multigrid convergence factors after 50 realizations of the random field.

\begin{tabular}{|c|c|c|}
\cline { 2 - 3 } \multicolumn{1}{c|}{} & $\lambda_{g}=0.3$ & $\lambda_{g}=0.1$ \\
\hline Grid1 & 0.19 & 0.20 \\
Grid2 & 0.19 & 0.21 \\
Grid3 & 0.20 & 0.29 \\
\hline
\end{tabular}

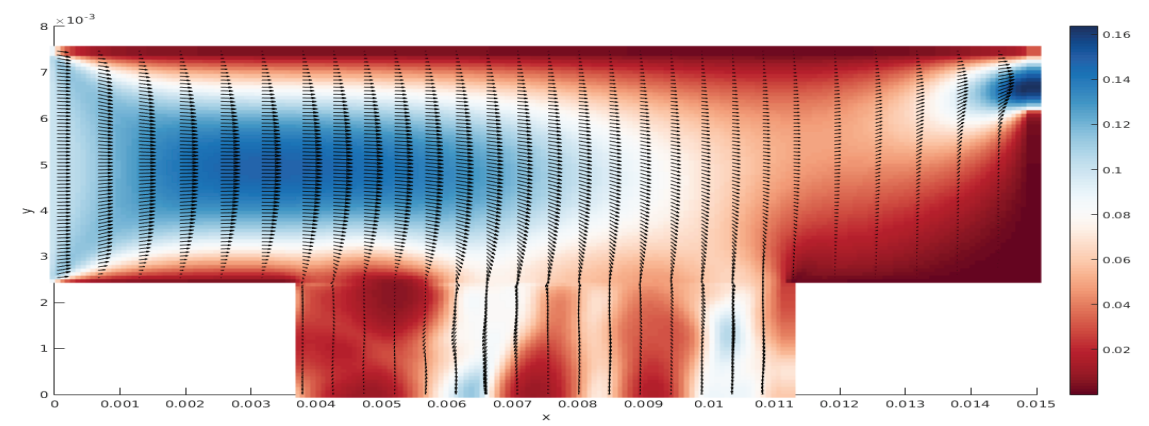

FIG. 14. $L_{2}$-norm of the velocity vectors over the cross-flow filtration domain with random distribution of hydraulic conductivity $K\left(\lambda_{g}=0.3\right)$.

Acknowledgment. We thank Prashant Kumar for his help and discussion regarding the MATLAB code for the heterogeneous test.

\section{REFERENCES}

[1] J. H. Adler, T. R. Benson, E. C. Cyr, S. P. Maclachlan, and R. S. Tuminaro, Monolithic multigrid methods for two-dimensional resistive magnetohydrodynamics, SIAM J. Sci. Comput., 38 (2016), pp. B1-B24, https://doi.org/10.1137/151006135.

[2] P. Angot, On the well-posed coupling between free fluid and porous viscous flows, Appl. Math. Lett., 24 (2011), pp. 803-810, https://doi.org/10.1016/j.aml.2010.07.008.

[3] O. Axelsson, Iterative Solution Methods, Cambridge University Press, Cambridge, UK, 1996.

[4] M. Benzi, G. Golub, And J. Liesen, Numerical solution of saddle point problems, Acta Numer., 14 (2005), pp. 1-137, https://doi.org/10.1017/S0962492904000212.

[5] D. Braess and R. Sarazin, An efficient smoother for the Stokes problem, Appl. Numer. Math., 23 (1997), pp. 3-19, https://doi.org/10.1016/S0168-9274(96)00059-1.

[6] A. BRAndt, Multi-level adaptive solutions to boundary-value problems, Math. Comp., 31 (1977), pp. 333-390.

[7] A. Brandt, Rigorous quantitative analysis of multigrid, I: Constant coefficients two-level cycle with $L_{2}$-norm, SIAM J. Numer. Anal., 31 (1994), pp. 1695-1730, https://doi.org/10.1137/ 0731087.

[8] A. Brandt and N. Dinar, Multigrid solutions to elliptic flow problems, in Numerical Methods for Partial Differential Equations, Academic Press, New York, London, 1979, pp. 53-147.

[9] A. Brandt and O. E. Livne, Multigrid Techniques: 1984 Guide with Applications to Fluid Dynamics, Classics Appl. Math. 67, SIAM, Philadelphia, 2011, https://doi.org/10.1137/1. 9781611970753.

[10] M. CAI, M. Mu, AND J. Xu, Preconditioning techniques for a mixed Stokes/Darcy model in porous media applications, J. Comput. Appl. Math., 233 (2009), pp. 346-355, https://doi. org/10.1016/j.cam.2009.07.029.

Copyright $@$ by SIAM. Unauthorized reproduction of this article is prohibited. 
[11] A. Caiazzo, V. John, and U. Wilbrandt, On classical iterative subdomain methods for the Stokes-Darcy problem, Comput. Geosci., 18 (2014), pp. 711-728, https://doi.org/10.1007/ s10596-014-9418-y.

[12] Y. Cao, M. Gunzburger, X. Hu, F. Hua, X. Wang, and W. Zhao, Finite element approximations for Stokes-Darcy flow with Beavers-Joseph interface conditions, SIAM J. Numer. Anal., 47 (2010), pp. 4239-4256, https://doi.org/10.1137/080731542.

[13] Y. CaO, M. Gunzburger, F. Hua, And X. Wang, Coupled Stokes-Darcy model with BeaversJoseph interface boundary condition, Commun. Math. Sci., 8 (2010), pp. 1-25.

[14] W. Chen, M. Gunzburger, F. Hua, And X. Wang, A parallel Robin-Robin domain decomposition method for the Stokes-Darcy system, SIAM J. Numer. Anal., 49 (2011), pp. 10641084, https://doi.org/10.1137/080740556.

[15] P. Chidyagwai, S. Ladenheim, and D. B. Szyld, Constraint preconditioning for the coupled Stokes-Darcy system, SIAM J. Sci. Comput., 38 (2016), pp. A668-A690, https://doi.org/ $10.1137 / 15 \mathrm{M} 1032156$.

[16] P. Chidyagwai And B. Rivière, Numerical modelling of coupled surface and subsurface flow systems, Adv. Water Resour., 33 (2010), pp. 92-105, https://doi.org/10.1016/j.advwatres. 2009.10.012.

[17] M. Discacciati, E. Miglio, and A. Quarteroni, Mathematical and numerical models for coupling surface and groundwater flows, Appl. Numer. Math., 43 (2002), pp. 57-74, https:// doi.org/10.1016/S0168-9274(02)00125-3.

[18] M. DiscaCciati And A. Quarteroni, Convergence analysis of a subdomain iterative method for the finite element approximation of the coupling of Stokes and Darcy equations, Comput. Vis. Sci., 6 (2004), pp. 93-103, https://doi.org/10.1007/s00791-003-0113-0.

[19] M. Discacciati and A. Quarteroni, Navier-Stokes/Darcy coupling: Modeling, analysis, and numerical approximation, Rev. Mat. Complut., 22 (2009), pp. 315-426.

[20] M. Discacciati, A. Quarteroni, And A. Valli, Robin-Robin domain decomposition methods for the Stokes-Darcy coupling, SIAM J. Numer. Anal., 45 (2007), pp. 1246-1268, https:// doi.org/10.1137/06065091X.

[21] F. Gaspar, F. Lisbona, and C. Oosterlee, A stabilized difference scheme for deformable porous media and its numerical resolution by multigrid methods, Comput. Vis. Sci., 11 (2008), pp. 67-76, https://doi.org/10.1007/s00791-007-0061-1.

[22] F. Gaspar, F. Lisbona, C. Oosterlee, and R. Wienands, A systematic comparison of coupled and distributive smoothing in multigrid for the poroelasticity system, Numer. Linear Algebra Appl., 11 (2004), pp. 93-113, https://doi.org/10.1002/nla.372.

[23] F. J. Gaspar, Y. Notay, C. W. Oosterlee, and C. Rodrigo, A simple and efficient segregated smoother for the discrete Stokes equations, SIAM J. Sci. Comput., 36 (2014), pp. A1187-A1206, https://doi.org/10.1137/130920630.

[24] N. S. Hanspal, A. N. Waghode, V. Nassehi, and R. J. Wakeman, Development of a predictive mathematical model for coupled Stokes/Darcy flows in cross-flow membrane filtration, Chem. Eng. J., 149 (2009), pp. 132-142, https://doi.org/10.1016/j.cej.2008.10.012.

[25] F. HARLOW AND J. Welch, Numerical calculation of time-dependent viscous incompressible flow of fluid with free surface, Phys. Fluids, 8 (1965), pp. 2182-2189, https://doi.org/10. 1063/1.1761178.

[26] M. Hoffmann, The Navier-Stokes-Darcy Problem, Bachelor's thesis, Weierstrass Institute, Berlin, Germany, 2013.

[27] W. JäGER AND A. Mikelić, On the interface boundary condition of Beavers, Joseph, and Saffman, SIAM J. Appl. Math., 60 (2000), pp. 1111-1127, https://doi.org/10.1137/ S003613999833678X.

[28] V. John AND L. ToBiska, Numerical performance of smoothers in coupled multigrid methods for the parallel solution of the incompressible Navier-Stokes equations, Internat. J. Numer. Methods Fluids, 33 (2000), pp. 453-473, https://doi.org/10.1002/1097-0363 (20000630)33:4<453::AID-FLD15>3.0.CO;2-0.

[29] I. P. Jones, Low Reynolds number flow past a porous spherical shell, Math. Proc. Cambridge Philos. Soc., 73 (1973), pp. 231-238, https://doi.org/10.1017/S0305004100047642.

[30] P. Kumar, C. W. Oosterlee, and R. P. Dwight, A multigrid multilevel Monte Carlo method using high-order finite-volume scheme for lognormal diffusion problems, Int. J. Uncertain. Quantif., 7 (2017), pp. 57-81, https://doi.org/10.1615/Int.J.UncertaintyQuantification. 2016018677.

[31] M. LaRIN AND A. Reusken, A comparative study of efficient iterative solvers for generalized Stokes equations, Numer. Linear Algebra Appl., 15 (2008), pp. 13-34, https://doi.org/10. 1002/nla.561.

Copyright (C) by SIAM. Unauthorized reproduction of this article is prohibited. 
[32] W. J. Layton, F. Schieweck, And I. Yotov, Coupling fluid flow with porous media flow, SIAM J. Numer. Anal., 40 (2003), pp. 2195-2218, https://doi.org/10.1137/ S0036142901392766.

[33] T. Levy and E. Sanchez-Palencia, On boundary conditions for fluid flow in porous media, Internat. J. Engrg. Sci., 13 (1975), pp. 923-940, https://doi.org/10.1016/ 0020-7225(75)90054-3.

[34] P. Luo, C. Rodrigo, F. J. Gaspar, and C. W. Oosterlee, On an Uzawa smoother in multigrid for poroelasticity equations, Numer. Linear Algebra Appl., 24 (2017), e2074, https://doi.org/10.1002/nla.2074.

[35] J.-F. Maitre, F. Musy, And P. Nigon, A fast solver for the Stokes equations using multigrid with a Uzawa smoother, in Advances in Multigrid Methods, Friedrich Vieweg \& Sohn, Braunschweig, Germany, 1985, pp. 77-83, https://doi.org/10.1007/978-3-663-14245-4_8.

[36] A. Márquez, S. Meddahi, And F.-J. Sayas, A decoupled preconditioning technique for a mixed Stokes-Darcy model, J. Sci. Comput., 57 (2013), pp. 174-192.

[37] C. Oosterlee And F. Gaspar, Multigrid relaxation methods for systems of saddle point type, Appl. Numer. Math., 58 (2008), pp. 1933-1950, https://doi.org/10.1016/j.apnum.2007.11. 014.

[38] M. Perić, R. Kessler, and G. Scheuerer, Comparison of finite-volume numerical methods with staggered and colocated grids, Comput. \& Fluids, 16 (1988), pp. 389-403, https://doi. org/10.1016/0045-7930(88)90024-2.

[39] A. Quarteroni and A. Valli, Domain Decomposition Methods for Partial Differential Equations, Numer. Math. Sci. Comput. Oxford Sci. Publ., The Clarendon Press, Oxford University Press, New York, 1999.

[40] B. Rivière And I. Yotov, Locally conservative coupling of Stokes and Darcy flows, SIAM J. Numer. Anal., 42 (2005), pp. 1959-1977, https://doi.org/10.1137/S0036142903427640.

[41] I. Rybak, J. Magiera, R. Helmig, and C. Rohde, Multirate time integration for coupled saturated/unsaturated porous medium and free flow systems, Comput. Geosci., 19 (2015), pp. 299-309, https://doi.org/10.1007/s10596-015-9469-8.

[42] P. Saffman, On the boundary condition at the surface of a porous medium, Stud. Appl. Math., 50 (1971), pp. 93-101, https://doi.org/10.1002/sapm197150293.

[43] V. Schmidt, ED., Stochastic Geometry, Spatial Statistics and Random Fields, Springer International, Cham, Switzerland, 2015, https://doi.org/10.1007/978-3-319-10064-7.

[44] B. Smith, P. Buørstad, And W. Gropp, Domain Decomposition: Parallel Multilevel Methods for Elliptic Partial Differential Equations, Cambridge University Press, Cambridge, UK, 2004.

[45] U. Trottenberg, C. W. Oosterlee, and A. Schüller, Multigrid, Academic Press, San Diego, 2001.

[46] S. VANKA, Block-implicit multigrid solution of Navier-Stokes equations in primitive variables, J. Comput. Phys., 65 (1986), pp. 138-158, https://doi.org/10.1016/0021-9991(86)90008-2.

[47] R. Wienands and W. Joppich, Practical Fourier Analysis for Multigrid Methods, Chapman \& Hall/CRC, Boca Raton, FL, 2005.

Copyright $@$ by SIAM. Unauthorized reproduction of this article is prohibited. 